Draft VERSion MAY 27, 2018

Preprint typeset using $\mathrm{LAT}_{\mathrm{E}} \mathrm{X}$ style emulateapj v. 08/22/09

\title{
SN 2006tf: PRECURSOR ERUPTIONS AND THE OPTICALLY THICK REGIME OF EXTREMELY LUMINOUS TYPE IIn SUPERNOVAE
}

\author{
Nathan Smith, Ryan Chornock, Weidong Li, Mohan Ganeshalingam, Jeffrey M. Silverman, \\ Ryan J. Foley, \& Alexei V. Filippenko \\ Department of Astronomy, University of California, Berkeley, CA 94720-3411; nathans@astro.berkeley.edu
} AND

AARON J. BARTH

Department of Physics and Astronomy, University of California, 4129 Frederick Reines Hall, Irvine, CA 92697-4575 Draft version May 27, 2018

\begin{abstract}
SN 2006tf is the third most luminous supernova (SN) discovered so far, after SN 2005ap and SN 2006gy. SN 2006tf is valuable because it provides a link between two regimes: (1) luminous type IIn supernovae powered by emission directly from interaction with circumstellar material (CSM), and (2) the most extremely luminous SNe where the CSM interaction is so optically thick that energy must diffuse out from an opaque shocked shell. As SN 2006tf evolves, it slowly transitions from the second to the first regime as the clumpy shell becomes more porous. This link suggests that the range in properties of the most luminous SNe is largely determined by the density and speed of hydrogen-rich material ejected shortly before they explode. The total energy radiated by SN 2006tf was at least $7 \times 10^{50} \mathrm{ergs}$. If the bulk of this luminosity came from the thermalization of shock kinetic energy, then the star needs to have ejected $\sim 18 \mathrm{M}_{\odot}$ in the $4-8 \mathrm{yr}$ before core collapse, and another $2-6 \mathrm{M}_{\odot}$ in the decades before that. A Type Ia explosion is therefore excluded. From the $\mathrm{H} \alpha$ emission-line profile, we derive a blast-wave speed of $2,000 \mathrm{~km} \mathrm{~s}^{-1}$ that does not decelerate, and from the narrow $\mathrm{P}$ Cygni absorption from pre-shock gas we deduce that the progenitor's wind speed was $\sim 190 \mathrm{~km} \mathrm{~s}^{-1}$. This is reminiscent of the wind speeds of luminous blue variables (LBVs), but not of red supergiants or Wolf-Rayet stars. We propose that like SN 2006gy, SN 2006tf marked the death of a very massive star that retained a hydrogen envelope until the end of its life, and suffered extreme LBV-like mass loss in the decades before it exploded.
\end{abstract}

Subject headings: circumstellar matter — stars: evolution — stars: mass loss — stars: winds, outflows — supernovae: individual (SN 2006tf)

\section{INTRODUCTION}

Massive stars that die as core-collapse supernovae (SNe) of Types II and Ib/c span a wide range in luminosity, but they are typically fainter than the standard thermonuclear Type Ia events that mark the deaths of lower-mass stars. More-luminous counterexamples are usually observed as SNe of Type IIn, exhibiting relatively narrow $\left(\sim 1000 \mathrm{~km} \mathrm{~s}^{-1}\right)$ emission lines of $\mathrm{H}$ in their spectra (Schlegel 1990; for a review of SN classification, see Filippenko 1997). These lines are generally attributed to shock interaction with dense circumstellar matter (CSM) rather than photospheric, high-velocity emission from the SN ejecta. They can show multiple components: a very narrow feature having typical widths up to a few hundred $\mathrm{km} \mathrm{s}^{-1}$ attributed to emission from pre-shock CSM shed by the progenitor, as well as broader components with widths of a few thousand $\mathrm{km} \mathrm{s}^{-1}$ arising from dense post-shock gas. We will refer to these two components as "narrow" and "intermediate-width" lines, respectively. By comparison, broad lines in SN ejecta typically have widths of $10,000-20,000 \mathrm{~km} \mathrm{~s}^{-1}$.

Sufficiently dense CSM can decelerate the blast wave and convert its bulk kinetic energy into X-rays and then visual radiation, thereby substantially increasing the bolometric luminosity of the SN. This is seen in luminous SNe IIn and II-L having very strong $\mathrm{H} \alpha$ emission, such as SNe 1979C, 1986J, 1988Z, 1994W, 1997cy, and others (Branch et al. 1981; Filippenko 1991, 1997; Leibundgut et al. 1991; Turatto et al. 1993; Sollerman et al. 1998; Chugai et al. 2004; Germany et al. 2000; Turatto et al. 2000; Benetti et al. 1998), as well as the "hybrid" Type Ia/IIn objects SN 2002ic (Hamuy et al. 2003; Wang et al. 2004; Wood-Vasey et al. 2004; Kotak et al. 2004; Benetti et al. 2006) and SN 2005gj (Prieto et al. 2007; Aldering et al. 2006). SNe 1997cy and 1999E may also be hybrid SNe of this type (Germany et al. 2000; Filippenko 2000; Rigon et al. 2003; Hamuy et al. 2003; Wood-Vasey et al. 2004).

Because it is the dense CSM that absorbs momentum and drains kinetic energy from the blast wave, moreluminous SNe IIn require progenitors with higher massloss rates or slower wind speeds. To account for some of the more-luminous SNe IIn, inferred progenitor massloss rates need to be of order $0.1 \mathrm{M}_{\odot} \mathrm{yr}^{-1}$ or higher (e.g., Chugai et al. 2004; Chugai \& Danziger 2003; Smith 2008). These extreme requirements point to episodic mass ejection reminiscent of the eruptions seen in $\eta$ Carinae and other luminous blue variables (LBVs), which have mass-loss rates of $0.01-1 \mathrm{M}_{\odot} \mathrm{yr}^{-1}$, far exceeding the limiting mass loss in line-driven stellar winds (Smith \& Owocki 2006; Owocki et al. 2004). In SN IIn progenitors, the reason for this extreme mass loss is not yet understood, but it must occur in the decades preceding core collapse because the blast wave can only reach radii 


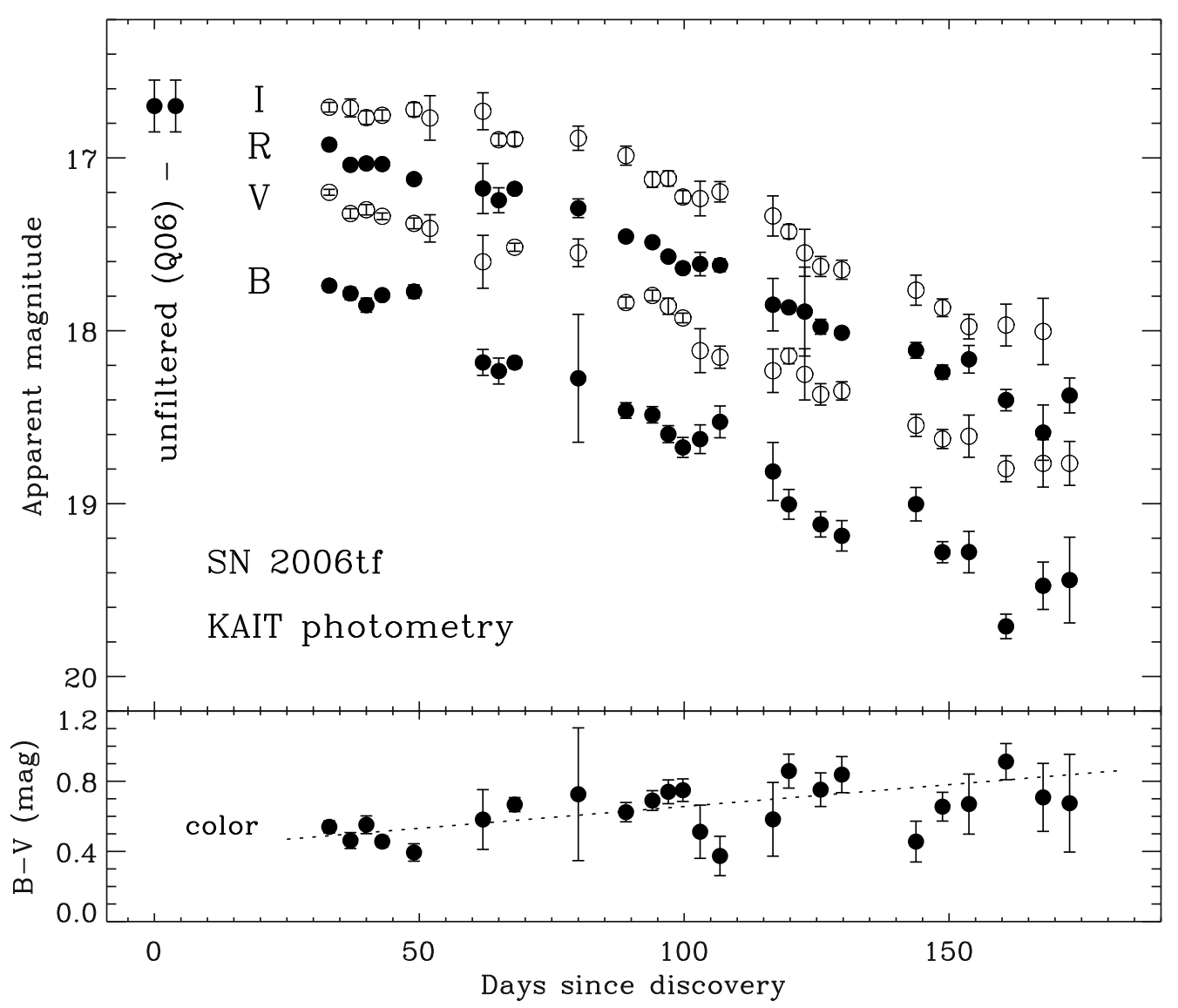

FIG. 1. - The top panel shows our BVRI light curves of SN 2006tf obtained with KAIT (see Table 1), and the bottom panel gives the apparent $B-V$ color. The dotted line illustrates a least-squares fit to the color change with a slope of $0.0025 \pm 0.0003$ mag $\mathrm{d}^{-1}$. Days since discovery are plotted because the explosion date is not known.

out to a few $10^{2}$ AU during early phases of these SNe.

Two recent events have pushed the limits on the required physical conditions, challenging the standard picture of CSM interaction: SN 2006gy (Ofek et al. 2007; Smith et al. 2007; Woosley et al. 2007; Smith \& McCray 2007) radiated more energy in visual light than any other known SN, and SN 2005ap (Quimby et al. 2007a) appears to have had the brightest peak absolute magnitude yet observed. To account for their extreme luminosities with CSM interaction, the required progenitor mass-loss rates are of order $1 \mathrm{M}_{\odot} \mathrm{yr}^{-1}$. The problem is that in SN 2006gy, the weak $\mathrm{H} \alpha$ and X-ray emission imply mass-loss rates a factor of $10^{2}-10^{4}$ smaller (Smith et al. 2007), while SN 2005ap shows no spectral signature of CSM interaction at all (i.e., it was not a SN IIn; Quimby et al. 2007a). One possible solution is that these SNe are indeed ultimately powered by CSM interaction, but this interaction occurs early in a highly opaque shell, forcing photons to diffuse out after the shock has already passed through it (Smith \& McCray 2007). An alternative view (Smith et al. 2007; Quimby et al. 2007a) is that these two SNe could be powered by radioactive decay from a large mass of ${ }^{56} \mathrm{Ni}$ synthesized in a pair-instability $\mathrm{SN}$ event (Barkat et al. 1967; Rakavy \& Shaviv 1967; Bond, Arnett, \& Carr 1984), but that hypothesis remains difficult to prove or rule out (see Smith et al. 2008b), partly because the decay luminosity would also need to diffuse out through a massive envelope.

In this paper we investigate yet another extremely lu- minous SN that is nearly as luminous as SNe 2006gy and 2005ap and has some similarity to them, but which exhibits signs of stronger CSM interaction in the spectrum.

SN 2006tf was discovered on 2006 Dec. 12 (UT dates are used throughout this paper) in the course of the Texas Supernova Search (Quimby et al. 2007b). In spectra obtained $\sim 10 \mathrm{~d}$ after discovery it showed Type IIn signatures. SN 2006tf is located 0.'2 east and 0.7 north of the center of an anonymous and faint galaxy with an apparent $R$ magnitude of 20.68 (Quimby et al. 2007b), for which the metallicity is not known. Its redshift, $z=0.074$, indicates a distance of $308 \mathrm{Mpc}$, assuming $\mathrm{H}_{0}=72 \mathrm{~km} \mathrm{~s}^{-1} \mathrm{Mpc}^{-1}$. At that distance, the discovery magnitude of about $m_{r}=16.7$, which was roughly constant for the first 2 weeks after discovery, makes the peak absolute magnitude about -20.7 , or roughly $1.6 \times 10^{10} \mathrm{~L}_{\odot}$ without correcting for extinction.

This extremely high luminosity makes SN 2006tf one of the most luminous SNe ever discovered. Among known SNe, it is surpassed only by SN 2006gy and SN 2005ap. It is more luminous than very bright or long-lasting SNe IIn with strong CSM interaction like SNe 1979C, 1988Z, 1994W, 1998S, and others, as well as the Type Ia/IIn "hybrid" objects mentioned earlier. Given its Type IIn spectrum, the extreme luminosity of SN 2006tf is likely to be powered by CSM interaction. With the lack of any Type IIn signatures in the spectrum of SN 2005ap (Quimby et al. 2007a), and the difficulty in reconciling the energy budget of SN 2006gy with its relatively weak 


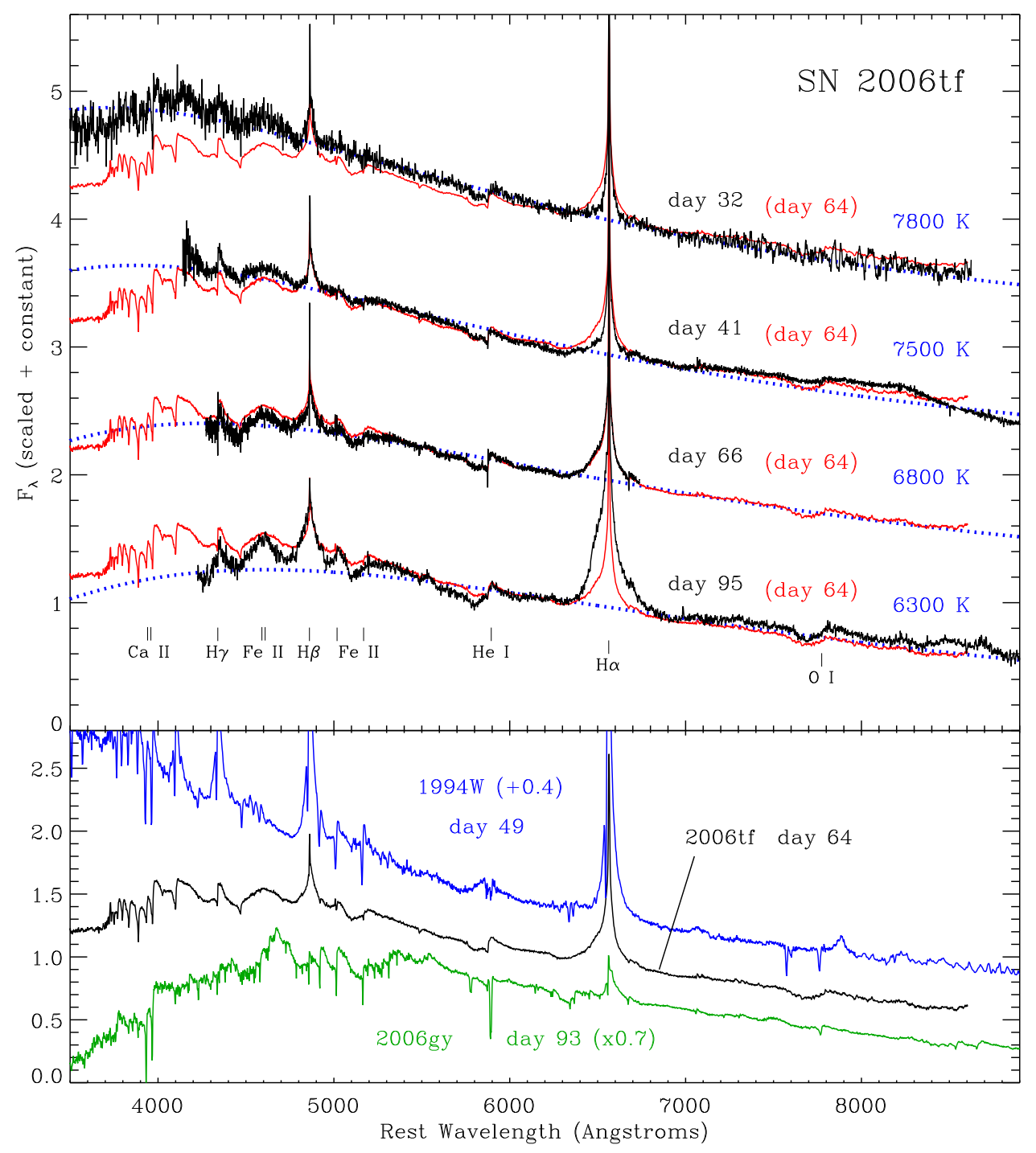

FIG. 2.- The top panel shows visual-wavelength spectra of SN 2006tf on days 32, 41, 66, and 95 since discovery, normalized to the red continuum level, and with constant offsets of $+3(\mathrm{~d} 32),+2(\mathrm{~d} 41)$, and +1 (d66). The day 64 spectrum (obtained for spectropolarimetry) is plotted in red several times for comparison with the other epochs. The dotted blue curves show representative black bodies for comparison at the temperatures indicated. At the bottom, the day 64 spectrum of SN 2006tf (black) is compared to the day 93 spectrum of SN $2006 \mathrm{gy}$ (green), dereddened assuming $E(B-V)=0.723 \mathrm{mag}$ (Smith et al. 2007), and to the day 49 spectrum of SN 1994W (blue) from our spectral database. All spectra of SN 2006tf have been corrected for $E(B-V)=0.027$ mag.

CSM interaction signatures (Smith et al. 2007, 2008b), the strong CSM signatures and comparably high luminosity of SN 2006tf are of significant interest. Therefore, in this paper we consider the photometric and spectroscopic evolution of SN 2006tf in detail.

We present our optical observations in $\S 2$. The light curve and bolometric luminosity are discussed in $\S 3$, and in $\S 4$ we describe the general spectral evolution. A detailed look at the $\mathrm{H} \alpha$ and $\mathrm{H} \beta$ emission-line profiles and their evolution is given in $\S 5$, including a late-time spectrum taken on day 445. Analysis of our spectropolarimetry of SN 2006tf is presented in $\S 6$. In $\S 7$ we estimate the most basic physical properties of SN 2006tf. Section 8 summarizes our main results, discussed in the context of an integrated picture of SN 2006tf and other SNe powered by CSM interaction.

\section{OBSERVATIONS}

We obtained optical photometry of SN 2006tf in BVRI using the $0.76 \mathrm{~m}$ Katzman Automatic Imaging Telescope (KAIT; Filippenko et al. 2001) at Lick Observatory. Flat fielding and bias subtraction were processed automatically. Galaxy subtraction and differential photometry were done using the KAIT pipeline (Ganeshalingam et al., in prep.). Template images of the galaxy were obtained on 2008 Jan. 18 with KAIT, more than 1 yr after discovery. Our late-time spectrum (see below) shows strong $\mathrm{H} \alpha$ emission and some faint continuum detected from the SN. Hence, there is some concern that our template image obtained on day 404 is partly contaminated by SN emission, rather than pure background galaxy emission. At this late time, we measure the magnitude at the position of the SN to be 20.1 in the $R$ band. Even if this late-time magnitude is dominated by SN light, the consequent oversubtraction would have caused us to overestimate the true $R$ magnitude by only $0.22 \mathrm{mag}$ 
TABLE 1

KAIT MAGNITUDES OF SN 2006 $\mathrm{TF}^{\mathrm{a}}$

\begin{tabular}{ccccccccc}
\hline \hline MJD & $B$ & err $_{B}$ & $V$ & err $_{V}$ & $R$ & err $_{R}$ & $I$ & err $_{I}$ \\
\hline 2454115.02 & 17.739 & 0.030 & 17.199 & 0.017 & 16.923 & 0.014 & 16.707 & 0.028 \\
2454118.99 & 17.784 & 0.036 & 17.322 & 0.028 & 17.040 & 0.021 & 16.711 & 0.052 \\
2454121.99 & 17.852 & 0.041 & 17.300 & 0.030 & 17.032 & 0.030 & 16.767 & 0.039 \\
2454125.00 & 17.794 & 0.025 & 17.338 & 0.019 & 17.036 & 0.014 & 16.754 & 0.031 \\
2454131.00 & 17.773 & 0.039 & 17.379 & 0.031 & 17.123 & 0.023 & 16.720 & 0.040 \\
2454133.94 & $\ldots$ & $\ldots$ & 17.408 & 0.079 & $\ldots$ & $\ldots$ & 16.769 & 0.129 \\
2454144.04 & 18.183 & 0.075 & 17.601 & 0.153 & 17.177 & 0.145 & 16.730 & 0.107 \\
2454146.97 & 18.233 & 0.075 & $\ldots$ & $\ldots$ & 17.245 & 0.072 & 16.895 & 0.035 \\
2454149.94 & 18.184 & 0.033 & 17.517 & 0.023 & 17.179 & 0.018 & 16.892 & 0.039 \\
2454161.98 & 18.275 & 0.370 & 17.549 & 0.080 & 17.291 & 0.054 & 16.886 & 0.070 \\
2454170.92 & 18.461 & 0.045 & 17.837 & 0.032 & 17.455 & 0.027 & 16.987 & 0.055 \\
2454175.90 & 18.486 & 0.047 & 17.796 & 0.031 & 17.488 & 0.028 & 17.125 & 0.045 \\
2454178.88 & 18.598 & 0.049 & 17.858 & 0.047 & 17.571 & 0.025 & 17.119 & 0.044 \\
2454181.86 & 18.675 & 0.058 & 17.926 & 0.028 & 17.638 & 0.031 & 17.226 & 0.037 \\
2454184.88 & 18.627 & 0.083 & 18.115 & 0.127 & 17.614 & 0.068 & 17.235 & 0.100 \\
2454188.86 & 18.527 & 0.092 & 18.153 & 0.064 & 17.621 & 0.037 & 17.196 & 0.059 \\
2454198.84 & 18.814 & 0.168 & 18.231 & 0.126 & 17.849 & 0.152 & 17.336 & 0.116 \\
2454201.80 & 19.004 & 0.086 & 18.146 & 0.045 & 17.865 & 0.029 & 17.426 & 0.041 \\
2454204.85 & $\ldots$ & $\ldots$ & 18.252 & 0.148 & 17.889 & 0.257 & 17.549 & 0.136 \\
2454207.82 & 19.120 & 0.073 & 18.368 & 0.062 & 17.977 & 0.043 & 17.628 & 0.058 \\
2454211.81 & 19.186 & 0.088 & 18.348 & 0.053 & 18.012 & 0.030 & 17.647 & 0.055 \\
2454225.81 & 19.003 & 0.097 & 18.547 & 0.064 & 18.113 & 0.046 & 17.765 & 0.087 \\
2454230.77 & 19.281 & 0.061 & 18.626 & 0.055 & 18.239 & 0.041 & 17.867 & 0.051 \\
2454235.79 & 19.280 & 0.120 & 18.610 & 0.122 & 18.165 & 0.080 & 17.976 & 0.071 \\
2454242.75 & 19.710 & 0.071 & 18.798 & 0.075 & 18.401 & 0.062 & 17.967 & 0.121 \\
2454249.71 & 19.475 & 0.137 & 18.767 & 0.137 & 18.589 & 0.160 & 18.004 & 0.192 \\
2454254.77 & 19.442 & 0.248 & 18.767 & 0.127 & 18.374 & 0.101 & $\cdots$ & $\cdots$ \\
& & & & & & & & \\
\hline
\end{tabular}

${ }^{\mathrm{a}}$ Photometric uncertainties are $1 \sigma$.

TABLE 2

Spectroscopic Observations of SN 2006TF

\begin{tabular}{|c|c|c|c|c|c|c|c|c|}
\hline Date & Day $^{\mathrm{a}}$ & Instrument & Range $_{\AA}^{b}$ & $\lambda / \Delta \lambda$ & $\begin{array}{c}\mathrm{EW}(\mathrm{H} \alpha) \\
\AA\end{array}$ & $\begin{array}{l}F(\mathrm{H} \alpha) \\
10^{-14} \mathrm{erg} \mathrm{s}^{-1} \mathrm{~cm}^{-2}\end{array}$ & $\mathrm{H} \alpha / \mathrm{H} \beta^{\mathrm{c}}$ & $\begin{array}{c}\mathrm{N} / \mathrm{T}^{\mathrm{d}} \\
\%\end{array}$ \\
\hline 2007 Jan. 13 & 32 & LRIS/Keck I & $3500-8600$ & 1000 & $-44(6)$ & $1.33(0.1)$ & 2.85 & 17 \\
\hline 2007 Jan. 22 & 41 & DEIMOS/Keck II & $4200-8900$ & 2000 & $-66(5)$ & $1.81(0.17)$ & 3.67 & 11.2 \\
\hline 2007 Feb. 14 & 64 & LRIS/Keck I (pol) & $3500-8600$ & 700 & $-72(5)$ & $2.19(0.16)$ & 3.48 & 9.0 \\
\hline 2007 Feb. 16 & 66 & DEIMOS/Keck II & $4300-6750$ & 8000 & $-82(7)$ & $1.90(0.2)$ & 3.79 & 8.1 \\
\hline 2007 Mar. 17 & 95 & DEIMOS/Keck II & $4200-8900$ & 2000 & $-194(11)$ & $3.39(0.24)$ & 4.84 & 3.4 \\
\hline 2008 Mar. 1 & 445 & ESI/Keck II & $4400-8800$ & 6000 & $-1035(90)$ & $0.69(0.34)$ & 11.6 & 3.6 \\
\hline
\end{tabular}

after day 150 , which is not much larger than the photometric uncertainty at that time.

Calibrations for the field were obtained on 8 photometric nights using both KAIT and the $1 \mathrm{~m}$ Nickel telescope at Lick Observatory. The uncertainty in our subtraction and photometry pipeline is estimated by injecting artificial stars with the same magnitude and point-spread function of the SN into regions of comparable complexity in the original KAIT images and recovering them. The final uncertainty is taken to be the scatter in recovering 20 artificial stars added in quadrature with the calibration error.

We take day zero to be MJD $=2,454,081.98$, the discovery date 2006 Dec. 12 (Quimby et al. 2007b). Since this is not the explosion date, there will be a source of continual ambiguity when comparing SN 2006tf to other $\mathrm{SNe}$. The explosion date is not known, but if the lightcurve evolution was similar to that of SN 2006gy, the explosion of SN 2006tf could conceivably have been long before the time of discovery. Figure 1 shows the KAIT magnitudes, which are listed in Table 1.

During the main peak of the light curve (within $200 \mathrm{~d}$ of discovery), we obtained visual-wavelength spectra of SN 2006tf on five separate dates at the Keck Observatory using the low resolution imaging spectrometer (LRIS; Oke et al. 1995) and the Deep Imaging Multi-Object Spectrograph (DEIMOS; Faber et al. 2003), with the observations summarized in Table 2. The epochs for these four spectra are days $32,41,64,66$, and 95 after discovery. The spectra were all obtained with the long slit oriented at the parallactic angle (Filippenko 1982), and were reduced using standard techniques. Wavelengths were corrected for redshift $z=0.074$ so that the narrow $\mathrm{H} \alpha$ emission line was at the proper rest wavelength. Given the very weak Na I D absorption and blue color, we made no correction for reddening and extinction beyond 
the relatively small Galactic values of $E(B-V)=0.027$ mag and $A_{R}=0.062 \mathrm{mag}$ (Schlegel et al. 1998). The resulting spectra are shown in Figure 2 ,

We also obtained one late-time optical spectrum on 2008 March 1, day 445 after discovery, using the Echelle Spectrograph and Imager (ESI; Sheinis et al. 2002) on the Keck II telescope (see Table 2). A 1200 s exposure was obtained in echellete mode, with the 0 .' 75 -wide slit oriented at position angle $45^{\circ}$. Because of relatively poor seeing of $\sim 1^{\prime \prime} .5$ (wider than the slit) and possible light cirrus during the night, the flux calibration is uncertain, and we conservatively adopt a factor of 2 uncertainty in absolute flux (i.e., $\mathrm{H} \alpha$ in Table 2). This is larger than the line-measurement uncertainty, and is hard to quantify. The late-time spectrum is discussed in $§ 5.4$.

Thus, we have two independent estimates of the latetime $R$ magnitude. As we mentioned earlier, our KAIT template image shows a magnitude of 20.1 , or $L=$ $7.5 \times 10^{8} \mathrm{~L}_{\odot}$, at the position of the SN. The true SN light could be substantially fainter than this if background galaxy light is important, but it cannot be much brighter (note that $\mathrm{H} \alpha$ also contaminates this measurement). From our late-time spectrum on day 445, in which we subtracted nearby background and still detected faint continuum emission from the $\mathrm{SN}$, we measure $F_{\lambda}=1.4 \mathrm{ergs} \mathrm{s}^{-1} \mathrm{~cm}^{-2} \AA^{-1}$ for the continuum level at red wavelengths, or an apparent $R$ magnitude of 20.3 . This corresponds to a late-time bolometric luminosity of $L=6.3 \times 10^{8} \mathrm{~L}_{\odot}$. Even though our uncertainty in the flux is a factor of two because of sky conditions, the agreement with the late-time KAIT magnitude to better precision than that is reassuring.

Of the five epochs of spectra during the first $100 \mathrm{~d}$, we obtained one epoch of spectropolarimetric observations of SN 2006tf on 2007 Feb. 14.54 (day 64) using the polarimeter unit ${ }^{1}$ of LRIS. The object was observed for $1000 \mathrm{~s}$ in each of the four rotation angles of the halfwave plate retarder. The total observed spectral range was $3350-9240 \AA$, and the 1. . 5 -wide slit gave a spectral resolution of $\sim 5 \AA$ in the blue and $\sim 9 \AA$ in the red. The spectropolarimetric reductions followed the procedure outlined by Miller et al. (1988) and implemented by Leonard et al. (2001). Observations of the polarized standard stars HD 19820 and HD 161056 (Schmidt et al. 1992) gave consistent results for the zero point of the instrument. Instrumental polarization was negligible as both of the observed null polarization standards showed $<0.1 \%$ polarization. Two foreground stars $(\mathrm{BD}+112491, \mathrm{BD}+122510)$, both within $20^{\prime}$ of the SN, were observed to constrain any potential Galactic component of interstellar polarization (ISP) due to dust grains along the line of sight. Both stars showed less than $0.1 \%$ polarization, indicating that the Galactic component of ISP is also negligible, consistent with the low dust column of $E(B-V)=0.027 \mathrm{mag}$ at the high Galactic latitude of SN 2006tf $\left(b=74^{\circ}\right)$.

\section{THE LIGHT CURVE AND BOLOMETRIC LUMINOSITY}

Our BVRI photometry of SN 2006tf obtained with KAIT is presented in Figure 1. Two features are immediately apparent. The first is the remarkably slow de-

\footnotetext{
1 See http://alamoana.keck.hawaii.edu/inst/lris/polarimeter/
} for the online polarimeter manual by Cohen (2005).

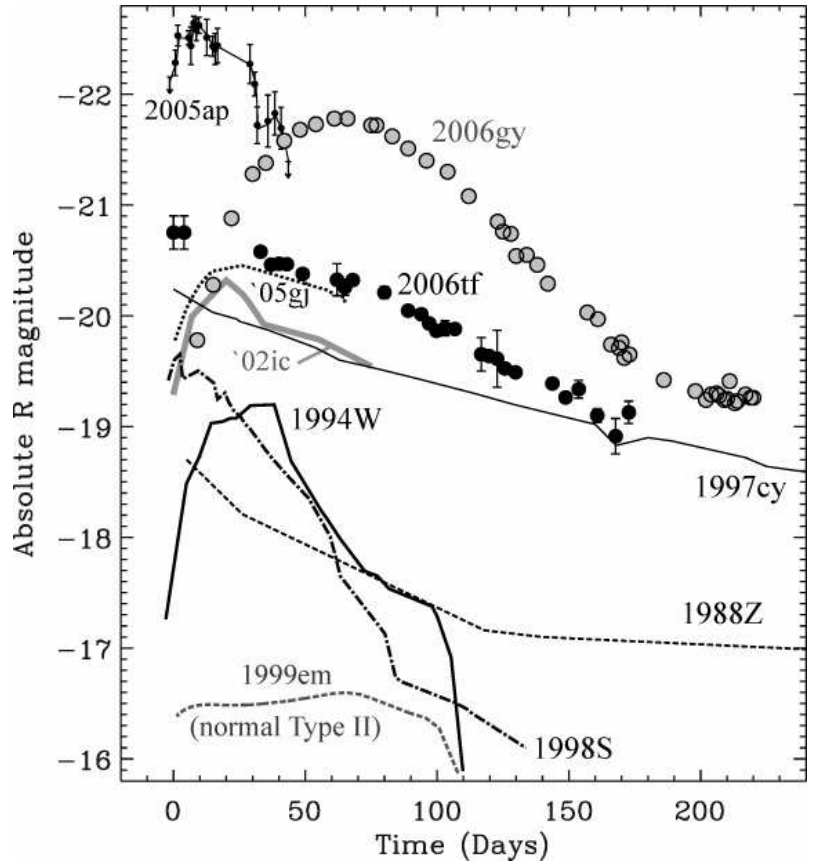

FIG. 3.- The absolute $R$-band light curve of SN 2006tf compared to that of several other luminous or strongly interacting SNe II and IIn. SN 2005ap is a Type II (not a IIn) from Quimby et al. (2007a) and SN 1999em is a normal Type II-P SN (Leonard et al. 2002). All others shown are Type IIn: SN 2006gy data are from Smith et al. (2007), SN 1994W data from Sollerman et al. (1998), SN 1988Z from Turatto et al. (1993), and SN 1998S from Fassia et al. (2000). SN 1997cy (Germany et al. 2000) is an extremely luminous SN IIn possibly associated with GRB 970514, or alternatively, a possible "hybrid" Type Ia/IIn like SNe 2002ic (Wood-Vasey et al. 2004; Hamuy et al. 2003) and 2005gj (Prieto et al. 2007) that are still difficult to understand (see also Benetti et al. 2006). When different values of $\mathrm{H}_{0}$ were used by the above authors, the absolute magnitudes have been adjusted to correspond to our adopted value of $72 \mathrm{~km} \mathrm{~s}^{-1} \mathrm{Mpc}^{-1}$.

cline in the light curve, with roughly $0.01 \mathrm{mag} \mathrm{d}^{-1}$ in the $R$ band, reminiscent of the slow evolution in SN 2006gy as well as the very slow decline in strongly interacting SNe IIn like SNe 1988Z, 1997cy, and 2005gj. The decline rate also matches the expected decay rate of ${ }^{56} \mathrm{Co}$, and this is discussed in more detail below. The second feature is the gradual change in color. The lower panel in Figure 1 shows the observed $B-V$ color, which gets redder at a rate of $\sim 0.0025 \pm 0.0003 \mathrm{mag} \mathrm{d}^{-1}$. This slow evolution to redder colors is in agreement with our spectra in Figure 2 in which the continuum slope gradually and monotonically evolves to lower temperatures. The relatively weak color changes, with temperatures that imply little or no bolometric correction, mean that we can take the absolute $R$ magnitude (corrected for small Galactic extinction) as a proxy for the bolometric magnitude, to compare SN 2006tf with other luminous SNe.

Thus, in Figure 3 we compare the absolute $R$ mag of SN 2006tf to that of several other notable SNe II/IIn. Near its peak at the time of discovery, SN 2006tf was the third most luminous SN observed so far after SN 2005ap and SN 2006gy. However, SNe 2005gj, $2002 \mathrm{ic}$, and $1997 \mathrm{cy}$ are not far behind. All of these are $\mathrm{SNe}$ II. Interestingly, though, all except the most luminous, SN 2005ap, were SNe IIn with prominent and relatively narrow $\mathrm{H} \alpha$ indicating strong CSM interaction. ${ }^{2}$

\footnotetext{
2 The true natures of SNe 2002ic, 2005gj, and 1997cy are still
} 
The strength of the SN IIn signature may be an important clue to the nature of the most luminous SNe: As we noted in a previous paper (Smith et al. 2007), while SN 2006gy was definitely a Type IIn with an $\mathrm{H} \alpha$ profile that indicated some CSM interaction, the strength of that interaction indicated by the $\mathrm{H} \alpha$ and X-ray luminosity seemed far too weak to be compatible with the power in continuum light. SN 2005ap was an even more extreme case, with no detectable sign of a narrow $\mathrm{H} \alpha$ feature generated by CSM interaction. SN 2006tf may be an important link, as its continuum luminosity and strong $\mathrm{H} \alpha$ are intermediate between these two extreme cases and other strongly interacting SNe. This will be a recurring theme in our investigation.

SN 2006tf was apparently discovered at, or perhaps somewhat after, its time of peak luminosity. The explosion date and the photometric behavior during the rise to maximum are not known. This should be kept in mind when interpreting Figure 3, because the translation of SN 2006tf on the time axis is necessarily quite uncertain. Judging by its similarity to SN 2006gy, for example, SN 2006tf could potentially be shifted as much as $50-70 \mathrm{~d}$ to the right to be more correctly compared with other SNe plotted here. Indeed, its high luminosity requires a large emitting radius of order 300 AU. That, combined with its maximum observed expansion speeds of order $7500 \mathrm{~km} \mathrm{~s}^{-1}$ seen in the broad component of the $\mathrm{H} \alpha$ line (see below), argue that it may have had quite a long rise time of order 50-100 d. Judging by SN 2002ic and SN 2005gj, both of which were caught during the rise to maximum, SN 2006tf should be shifted to the right in Figure 3 by at least $20 \mathrm{~d}$.

In any case, SN 2006tf clearly has an extremely high peak luminosity and a very long duration, with no sign, as yet, of a sharp decline like that of SNe 1994W or 2005ap (Fig. 3). As such, it demands an explanation for its high fluence that far exceeds that of normal corecollapse SNe. In general, such longevity at high luminosity implicates a large mass of emitting material, as we shall see below. Although less extreme, the energy demands of SN 2006tf rival those of SN 2006gy, and considerations for the possible energy source are similar. More in-depth considerations of the possible energy sources and associated difficulties can be found in our previous papers on SN 2006gy (Smith et al. 2007, 2008b; Smith \& McCray 2007). The two sources of luminosity we consider here are ${ }^{56} \mathrm{Co}$ decay from a large initial mass of ${ }^{56} \mathrm{Ni}$, or shock-deposited kinetic energy diffusing out of an opaque envelope after being thermalized and converted to visual light.

\subsection{Radioactive Decay?}

One possible source for a long lasting, extremely high SN luminosity is radioactive decay from a large initial mass of ${ }^{56} \mathrm{Ni}$. This might be synthesized in a "hypernova" or collapsar as has been proposed in the case of SN 1997cy where $2.3-2.6 \mathrm{M}_{\odot}$ of ${ }^{56} \mathrm{Ni}$ was derived (Germany et al. 2000; Turatto et al. 2000); in that case, it is interesting that the light curve of SN 1997cy is quite similar to that of SN 2006tf. Alternatively, a large mass of ${ }^{56} \mathrm{Ni}$ could potentially be synthesized in a pair-instability

controversial: SNe Ia exploding in dense $\mathrm{H}$ envelopes or perhaps the deaths of massive stars; see Benetti et al. (2006).

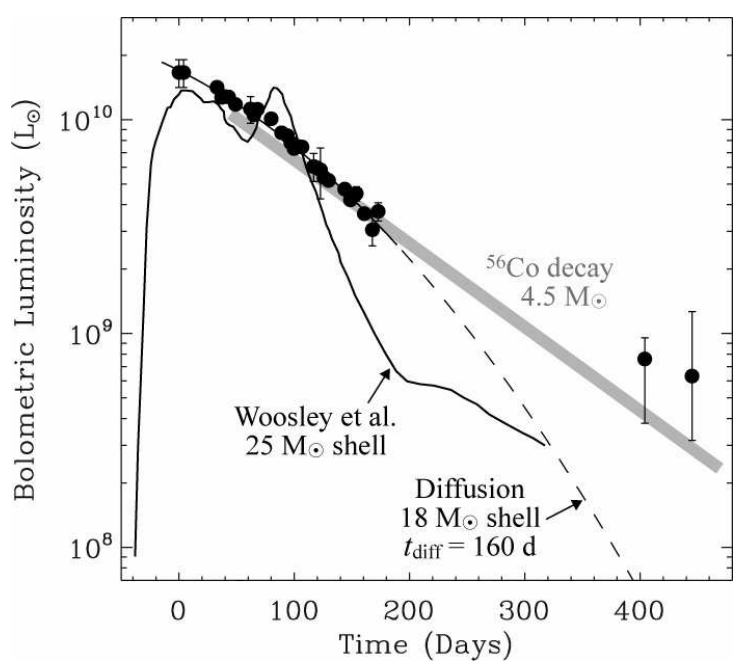

FIG. 4.- The absolute $R$ magnitudes of SN 2006tf from Figure 3 are converted to luminosities in $\mathrm{L}_{\odot}$; we also include the uncertain late-time magnitudes mentioned in $\S 2$. These are compared to a simple photon diffusion model (Smith \& McCray 2007) and the decline rate of ${ }^{56} \mathrm{Co}$ decay. The diffusion model (long dashes) gives an acceptable fit to the light curve for a diffusion timescale $t_{\text {diff }}=160 \mathrm{~d}$ and a CSM shell of $\sim 18 \mathrm{M}_{\odot}$, while radioactive decay (thick gray line) would require $\sim 4.5 \mathrm{M}_{\odot}$ of ${ }^{56} \mathrm{Ni}$ to power the same luminosity (see text). We also show a CSM interaction model for a progenitor star of $110 \mathrm{M} \odot$ and a circumstellar envelope mass of $\sim 25 \mathrm{M}_{\odot}$ (Woosley et al. 2007).

SN explosion (Bond et al. 1984; Barkat et al. 1967; Rakavy \& Shaviv 1967), as had been noted as a possibility for SN 2006gy (Smith et al. 2007) and SN 2005ap (Quimby et al. 2007a).

Figure 4 shows the bolometric luminosity light curve of SN 2006tf, derived from the absolute $R$ magnitude (adopting $A_{R}=0.062 \mathrm{mag}$ ). The thick gray line shows the ${ }^{56} \mathrm{Co}$ luminosity and decay rate one would expect from an initial ${ }^{56} \mathrm{Ni}$ mass of about $4.5 \mathrm{M}_{\odot} \cdot{ }^{3}$ Although it does not match the light curve precisely, radioactive decay does reproduce the average decline rate of SN 2006tf quite well. It gives as good an account of the light curve as the $2.3-2.6 \mathrm{M}_{\odot}$ of ${ }^{56} \mathrm{Ni}$ proposed in the case of SN 1997cy (Germany et al. 2000; Turatto et al. 2000).

Furthermore, radioactive decay makes a clear prediction for the late-time luminosity: it should follow the ${ }^{56}$ Co decay rate. As we noted earlier, on day 404, a source at the position of SN 2006tf had an $R$ magnitude of roughly 20.1 (or somewhat fainter) in the template image that we used to subtract background light and calibrate our KAIT photometry in Figure 1. Also, from the continuum level in a late-time spectrum obtained on day 445 , we estimate an apparent $R$ magnitude of $20.3 \pm 0.75$, as noted above. The late-time luminosity predicted by the same ${ }^{56} \mathrm{Co}$ decay from the earlier light curve is roughly consistent with these late-time measurements. The decay model actually underpredicts the luminosity, but so do the CSM interaction models in Figure 4, and hence any model requires us to invoke some additional CSM interaction at late times.

Thus, we cannot easily discount ${ }^{56} \mathrm{Co}$ decay as a potentially significant source of luminosity for SN 2006tf,

\footnotetext{
3 The necessary ${ }^{56} \mathrm{Ni}$ mass is very uncertain. It could be $7 \mathrm{M} \odot$ if our adopted $t=0$ is actually $\sim 50 \mathrm{~d}$ after the explosion date, or it could be arbitrarily less if CSM interaction contributes some substantial fraction of the luminosity.
} 
especially if some portion of the luminosity is supplied by CSM interaction as indicated by the spectral characteristics. This was also the case for SN 1997cy and SN 2006gy. Although we cannot confidently rule out radioactive decay, it seems more likely to us that SN 2006tf is dominated by extreme CSM interaction.

\subsection{Photon Diffusion From a Massive Shocked Envelope?}

A better match to the main light-curve peak of SN 2006tf is attained with an opaque shell-shocked model like that which Smith \& McCray (2007) proposed for the main light-curve peak of SN 2006gy (Fig. 4). In such a model, the extreme luminosity is a product of the large initial radius of an optically thick CSM ejecta envelope, throughout which shock kinetic energy has been thermalized efficiently. If the envelope is highly opaque, radiation must diffuse out rather than being emitted directly by the post-shock cooling zone. This is true no matter what the ultimate source of energy deposition is, whether it be shock energy or radioactive decay, or both.

The main ingredients that determine the shape of the diffusion light curve are the characteristic time for photon diffusion, $t_{\text {diff }}=\tau R / c$, and the expansion time, $t_{\exp }$ (see Smith \& McCray 2007; Falk \& Arnett 1973, 1977). These can be expressed as $t_{\text {diff }} \approx 23\left(M / R_{15}\right)$ d, with $M$ being the envelope mass in $\mathrm{M}_{\odot}$ and $R_{15}$ being the characteristic initial shell radius in units of $10^{15} \mathrm{~cm}$. The expansion timescale is just $t_{\exp } \approx\left(\Delta R / v_{\exp }\right)$ for constant expansion speed. Our model (dashed curve in Fig. 4) is a simple analytic model with $t_{\text {diff }}=160 \mathrm{~d}, R_{15}=2.7$, and $M=18 \mathrm{M}_{\odot}$. For comparison, Figure 4 also shows a onedimensional (1-D) numerical model of a similar physical situation from Woosley et al. (2007), with a $25 \mathrm{M}_{\odot}$ shell ejected by a $110 \mathrm{M}_{\odot}$ progenitor star about $5 \mathrm{yr}$ before the SN. This model was intended for SN 2006gy, but seems even more applicable to SN 2006tf. ${ }^{4}$

The diffusion curve in Figure 4 provides an excellent fit to the observed data by adopting $t_{\text {diff }}=t_{\text {exp }}=160 \mathrm{~d}$ (maximum luminosity for a given mass is achieved when these two are equal, minimizing adiabatic losses). If the characteristic expansion speed of the blast wave is about $2,000 \mathrm{~km} \mathrm{~s}^{-1}$, as indicated by the intermediate-width $\mathrm{H} \alpha$ emission (see below), then $R_{15}=2.7$ or about $180 \mathrm{AU}$ for $t_{\exp }=160 \mathrm{~d}$. This is the initial radius of the shocked ejecta envelope, which will grow with time. Thus, it is in approximate agreement with the somewhat larger radius of $\sim 300$ AU that we would nominally expect for $\mathrm{a} \sim 7800 \mathrm{~K}$ black body emitting the peak luminosity of SN 2006tf (see below). For $t_{\text {diff }}=160 \mathrm{~d}$, the required envelope mass is $\sim 18 \mathrm{M}_{\odot}$. These values are similar to, but even more extreme than, those of SN 2006gy: $t_{\text {diff }}=70 \mathrm{~d}, R=160 \mathrm{AU}$, and $M=10 \mathrm{M}_{\odot}$, respectively (Smith \& McCray 2007). As in that case, this radius is far too large to be the actual hydrostatic radius of a red supergiant progenitor, and the CSM-wind expansion speed of $190 \mathrm{~km} \mathrm{~s}^{-1}$ noted earlier is much too fast for that interpretation as well. Instead, this large radius probably represents the pseudo-photosphere of an opaque pre-SN ejecta shell that is not bound to the star, having been

\footnotetext{
4 Woosley et al. (2007) note that the peaks in the light curve may be an artifact of their 1-D models, which they expect would be smoothed out in reality.
}

ejected $\sim 4$ yr prior to the $\mathrm{SN}$ if it has been moving at $\sim 190 \mathrm{~km} \mathrm{~s}^{-1}$. Containing $18 \mathrm{M}_{\odot}$ within a radius of 180 AU would give at least $\tau \approx 400$.

In this model, a self-consistent explanation for the lower peak luminosity and slower decline rate of SN 2006tf as compared to SN 2006gy is that the forward shock of SN 2006tf has a slower expansion speed of $2,000 \mathrm{~km} \mathrm{~s}^{-1}$, compared to $4,000 \mathrm{~km} \mathrm{~s}^{-1}$ for SN 2006gy (Smith et al. 2007). The slower expansion speed causes a lower peak luminosity and longer duration, because the same amount of thermal energy will take a longer time to leak out of the envelope and the radius is smaller at a given time after explosion.

Although a simple model like this can fit the main light-curve peak, it is not necessarily a unique explanation, and both types of CSM interaction models (Smith \& McCray 2007; Woosley et al. 2007) fall short of the observed late-time luminosity (Fig. 4). As with SN 2006gy, there must be some additional contribution of emission directly from the ongoing, more optically thin CSM interaction region in order to explain the strong $\mathrm{H} \alpha$ emission. This occurs subsequent to the shock wave passing through the massive opaque shell, before which time the post-shock $\mathrm{H} \alpha$ emission would not be seen.

\subsection{Total Radiated Energy}

Regardless of which interpretation is correct, the photon diffusion model in Figure 4 gives an accurate phenomenological fit to the bolometric luminosity, so it can be used to measure the total radiated energy. Integrating this curve in Figure 4 from day 0 to 180, we find that the total energy radiated in visual light during the time of our photometric monitoring was $(6.2 \pm 0.3) \times 10^{50}$ ergs, if we assume no bolometric correction to the absolute $R$ magnitude. If we include the late-time tail, this value rises to about $7.0 \times 10^{50} \mathrm{ergs}$, and it could be increased even further if the time of discovery occurred significantly after the actual time of explosion. This is likely to be the case if SN 2006tf had a slow rise time comparable to SN 2006gy, or even a faster rise akin to SNe 2002ic and 2005gj, as we noted earlier.

It is interesting to note that an $18 \mathrm{M}_{\odot}$ shell moving at $2000 \mathrm{~km} \mathrm{~s}^{-1}$ contains the same amount of kinetic energy of about $7 \times 10^{50}$ ergs. Rough equipartition of thermal and kinetic energy is not surprising in this model (see Smith \& McCray 2007).

In any case, the total amount of energy radiated in visual light is almost $10^{51}$ ergs, or about half that of SN 2006gy (Smith et al. 2007). This amount of radiated energy must drain the reservoir of total available kinetic energy. Since the SN showed no sign of deceleration, this suggests either (1) that the CSM interaction we see now is not the major power source for the light curve, occurring subsequent to the shock passing through the opaque envelope mentioned earlier, or (2) that SN 2006tf marked an unusually energetic explosion well in excess of the canonical kinetic energy of a SN, as was the case with SN 2006gy (see Smith et al. 2007 for further discussion).

\section{THE VISUAL-WAVELENGTH SPECTRUM AND CONTINUUM}

The top panel in Figure 2 shows our visual-wavelength spectra of SN 2006tf on the four dates during the decline from the main peak of the light curve, normalized to the 
level of the red continuum flux and offset by constant values as noted in the caption. The epoch on day 64 is repeated in red for comparison to the other 4 epochs (this epoch has the highest signal-to-noise ratio because it was obtained for spectropolarimetry; see $\S 6$ ). These spectra have been corrected for Galactic reddening of $E(B-V)=0.027$ mag.

One of the most striking changes with time is in the relative strength of $\mathrm{H} \alpha$, to be discussed in detail in the following section. Here we focus mainly on the continuum shape and overall properties of the spectra.

In general, Figure 2 reinforces our earlier conclusion from photometry that SN 2006tf shows mild change in its color and continuum shape during the first $100 \mathrm{~d}$ after discovery, with correspondingly little evolution in the character of the spectrum. Matching the continuum with black bodies (dotted blue curves in the top panel of Fig. 2), the characteristic temperature drops monotonically from $7800 \mathrm{~K}$ to $6300 \mathrm{~K}$ between days 32 and 95 , consistent with the steady change in $B-V$ (Fig. 11).

Interestingly, the slow drop in temperature and characteristic line-blanketing features shortward of $4500 \AA$ are atypical for strongly interacting SNe IIn, which tend to show very blue continua at higher temperatures that remain nearly constant if the blast-wave speed is constant. Instead, the slow decline in temperatures around $7000 \mathrm{~K}$ and pronounced line blanketing are more reminiscent of normal SNe II-P, where photons are diffusing out of the expanding SN ejecta through which the recombination photosphere is receding. Since SN 2006tf is far too luminous for this emission to arise in normal SN ejecta, this is another argument favoring the shell-shocked photon diffusion model that we mentioned earlier, and the sustained high luminosity means that this shell must be very massive. SN 2006tf is distinguished from SNe II-P, however, in that its observed constant expansion speed indicates that this process must occur in a thin expanding shell rather than the geometrically thick envelope with a large velocity gradient in SNe II-P.

In the bottom panel of Figure 2 we compare the spectrum of SN 2006tf (black) to that of two other wellstudied SNe IIn from our spectral database. SN 1994W (blue) is a case where the spectrum and luminosity are dominated by ongoing CSM interaction with a contribution from diffusion of radiation from the SN ejecta or a shocked opaque envelope (Chugai et al. 2004), whereas in SN 2006gy (green), most of the continuum luminosity arises from photon diffusion from a previously shocked opaque shell (Smith \& McCray 2007). ${ }^{5}$ The continuum shape of SN 2006gy matches that of SN 2006tf quite well, as noted by Smith et al. (2007), but the $\mathrm{H} \alpha$ line of SN 2006tf is much stronger relative to its continuum. Taking into account both the continuum shape and the strong Balmer-line emission, we find that a linear combination of the spectra of both SN 1994W and SN 2006gy gives a suitable approximation to SN 2006tf. This suggests that ongoing CSM interaction, as compared to diffusion, contributes a larger fraction of the observed lu-

\footnotetext{
5 The fact that we are comparing a day 64 spectrum of SN 2006tf to a day 93 spectrum of SN 2006 gy is not necessarily cause for concern, since one is the date since discovery, and the other is the much longer time since the presumed explosion date. They represent comparable times after peak luminosity.
}

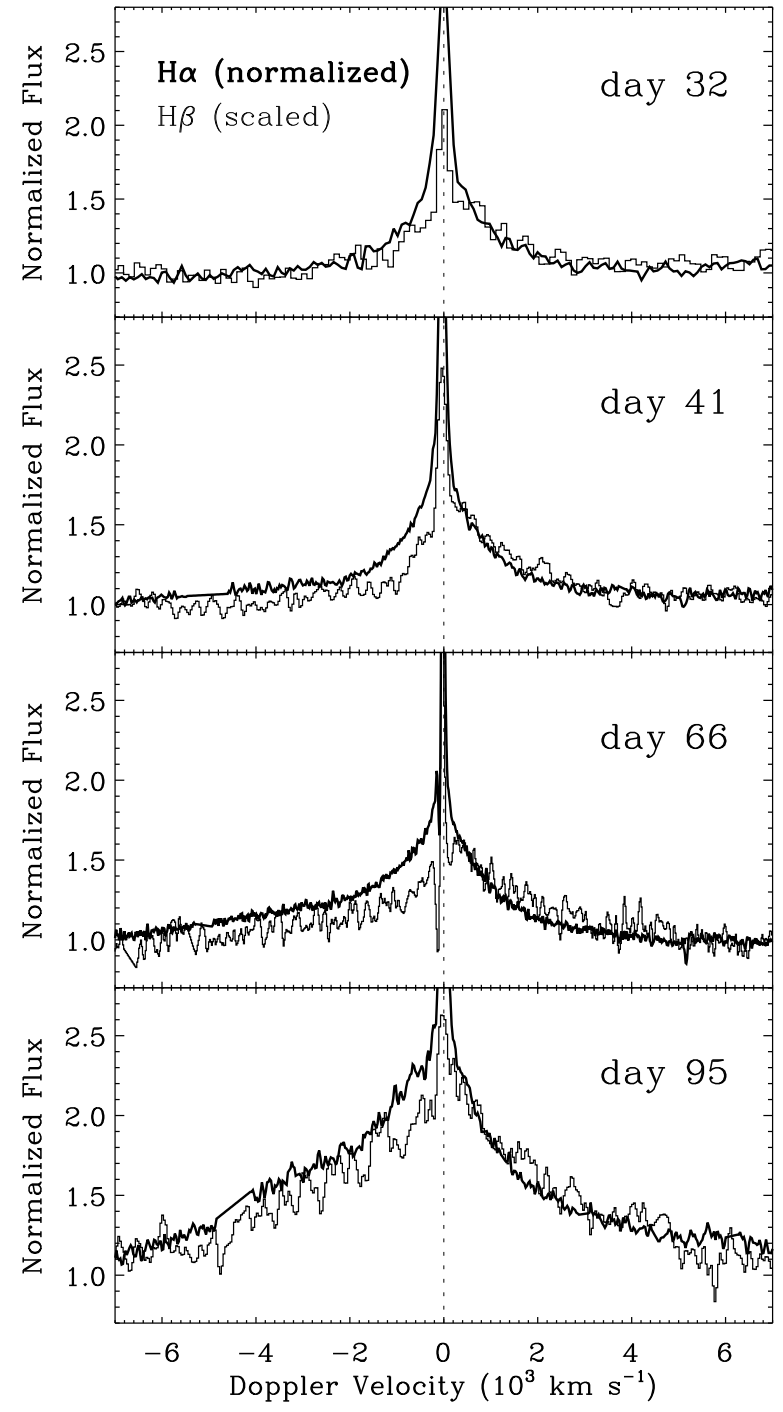

FIG. 5.- Observed line profiles of $\mathrm{H} \alpha$ and $\mathrm{H} \beta$ in SN 2006tf. $\mathrm{H} \alpha$ has been normalized with a fit to the observed continuum level. $\mathrm{H} \beta$ for each date is normalized and then scaled arbitrarily for comparison with the $\mathrm{H} \alpha$ profile. Day 64 is not shown here or in the next few figures because it is only $2 \mathrm{~d}$ before day 66 and has lower spectral resolution.

minosity in SN 2006tf than in SN 2006gy. Furthermore, the increase in the relative strength of the broad component of $\mathrm{H} \alpha$ with time implies that CSM interaction contributes a larger fraction of the luminosity at late times as the continuum source fades. This makes a strong case that SN 2006tf is a valuable example of the transition region between opaque shocked shells, like in SNe 2006gy and 2005ap, and progenitors with lower mass-loss rates where CSM-interaction is seen directly, like in SN 1988Z.

Finally, we note that aside from its $\mathrm{H} \alpha$ emission, the spectrum of SN 2006tf does not resemble the "hybrid" SNe IIn/Ia objects such as SNe 2002ic and 2005gj at any epoch, even though their light-curve shapes and absolute magnitudes are similar at early times. We detect no evidence for features from an underlying SN Ia spectrum. Additionally, the required envelope mass of $18 \mathrm{M}_{\odot}$ and total radiated energy of almost $10^{51}$ ergs make a SN Iapowered interpretation unlikely for SN 2006tf. 


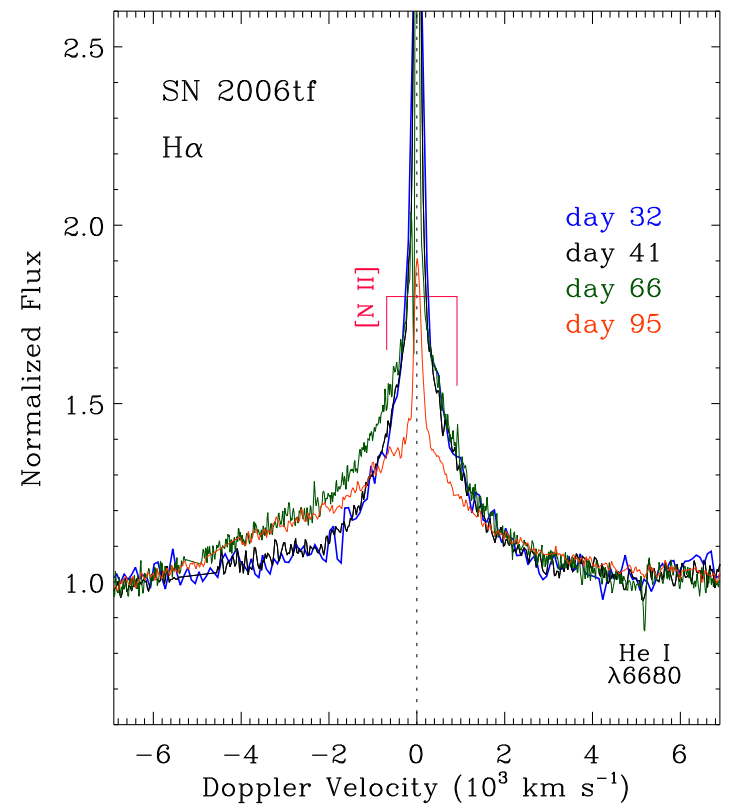

Fig. 6.- Observed $\mathrm{H} \alpha$ profiles on the four dates indicated, normalized and superposed over one another. The first three dates are plotted without further scaling, but the last one on day 95 has been adjusted by dividing the normalized intensity by 3 and adding 0.66 to compare the line shape with the other dates. Velocities at which [N II] $\lambda \lambda 6548,6583$ would be observed are indicated.

\section{EVOLUTION OF $\mathrm{H} \alpha$ AND $\mathrm{H} \beta$ EMISSION}

Figure 5 shows both $\mathrm{H} \alpha$ and $\mathrm{H} \beta$ at all four epochs, with the $\mathrm{H} \alpha$ flux normalized to the nearby continuum, and the $\mathrm{H} \beta$ profile scaled for comparison. It is evident that $\mathrm{H} \beta$ is more asymmetric than $\mathrm{H} \alpha$, showing a persistent deficit in the blueshifted emission wing at all epochs after day 32 , due to high optical depths and the stronger selfabsorption in $\mathrm{H} \beta$.

The intermediate-width component of $\mathrm{H} \alpha$ at roughly $\pm 2000 \mathrm{~km} \mathrm{~s}^{-1}$ is discussed in $\S 5.1$, while broad emission at relatively high speeds is discussed in $\S 5.2$. In $\S 5.3$ we describe the profiles of the narrowest component from the unshocked CSM, in $\$ 5.4$ we present the late-time spectrum, and in $\$ 5.5$ we discuss the behavior of the line luminosity with time.

\subsection{Post-Shock $H \alpha$ and $H \beta$ Profiles}

Figure 6] shows the intermediate-width $\mathrm{H} \alpha$ profile on days $32,41,66$, and 95 . This component at $\pm 2000 \mathrm{~km}$ $\mathrm{s}^{-1}$ dominates the appearance of the spectrum and accounts for most of the flux in the $\mathrm{H} \alpha$ line. The $\mathrm{H} \alpha$ profile appears to be identical on days 32 and 41, and the red wing of the line maintains the same shape at all epochs. However, we see a stronger, broad, blueshifted wing in the day 66 and 95 spectra. This enhanced blueshifted emission wing makes the line appear asymmetric. However, we suspect that it is not related directly to the emission from post-shock gas, but instead is due to an underlying broad component that is seen as the shell becomes more effectively optically thin, as discussed in the next section. Ignoring this broad blueshifted wing for now, the intermediate-width component of $\mathrm{H} \alpha$ is symmetric and centrally peaked in SN 2006tf, typical of the $\mathrm{H} \alpha$ profiles that define SNe IIn.

The speed of the blast wave expanding into the CSM can be inferred most clearly from the width and shape

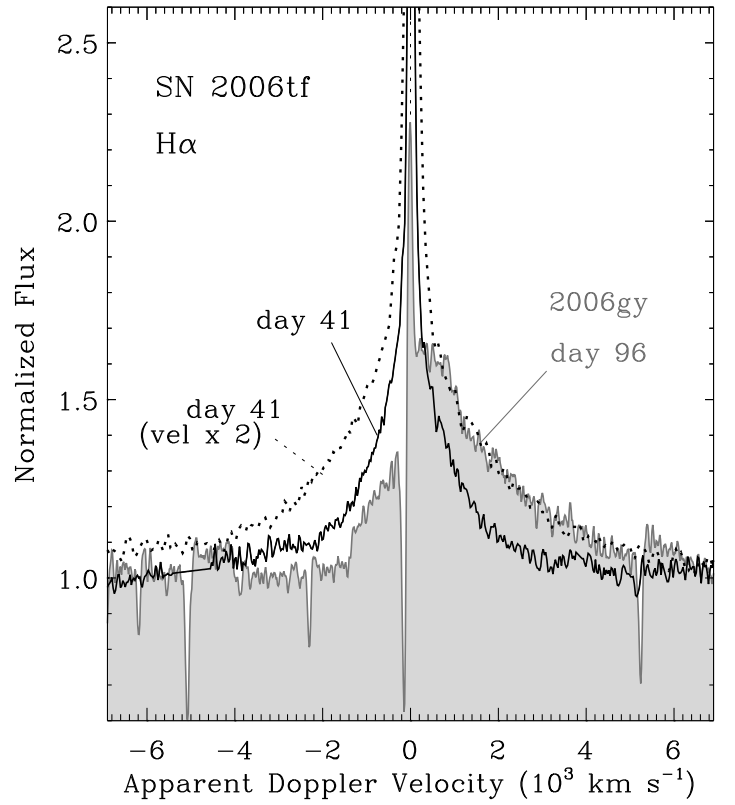

FIG. 7.- Same as Figure 6 but comparing the day 41 spectrum of SN 2006tf (solid black) to the day $96 \mathrm{H} \alpha$ profile of SN 2006gy (shaded gray, from Smith et al. 2007; the flux has been scaled upward because the $\mathrm{H} \alpha$ emission of SN 2006gy is much weaker relative to its continuum level). The profile of SN 2006gy is noticeably broader than that of SN 2006tf. If we expand the velocity scale of SN 2006tf by a factor of 2 (dashed line), then the red side of the broad $\mathrm{H} \alpha$ profile matches that of SN 2006gy quite well. This suggests that the blast-wave speed of SN $2006 \mathrm{tf}$ is roughly half as fast as that of SN 2006gy.

of this intermediate-width $\mathrm{H} \alpha$ profile. Figure 7 shows the $\mathrm{H} \alpha$ profile of SN $2006 \mathrm{tf}$ on day 41, when it is most symmetric, and compares it to the observed broad $\mathrm{H} \alpha$ profile of SN 2006gy from Smith et al. (2007). The reason this is useful is because the broad P Cygni absorption feature in SN 2006gy has a sharp blue edge at $-4,000 \mathrm{~km}$ $\mathrm{s}^{-1}$ that provides a good measure of the likely speed of the blast wave (Smith et al. 2007), whereas the profile of SN 2006tf has no sharp blue edge to provide a convenient measure of its true speed.

The narrower $\mathrm{H} \alpha$ profile of SN 2006tf is an obvious clue that its blast-wave speed is significantly slower than that of SN 2006gy. If we artificially stretch the velocity scale of the day $41 \mathrm{H} \alpha$ profile of SN 2006tf by a factor of 2, shown by the dotted line in Figure 7, then the red wing of the line matches that of SN 2006gy almost perfectly. (A comparison on the blue side does not work because of P Cygni absorption in SN 2006gy.) This provides a strong case that the blast-wave speed in SN 2006tf is about half that of SN 2006gy. Therefore, we adopt $2,000 \mathrm{~km} \mathrm{~s}^{-1}$ as the characteristic blast-wave speed for SN 2006tf, defining the speed at which the CSM is swept up by the forward shock. This also matches the full width at half-maximum intensity (FWHM) of the intermediate component of the $\mathrm{H} \alpha$ line in SN 2006tf. Judging from the constant red wing (Fig. 6), this speed remains unchanged for at least the first $100 \mathrm{~d}$, during a time when the $\mathrm{SN}$ is radiating away $\sim 10^{51}$ ergs in visual light.

\subsection{Underlying Broad Component}

As noted earlier, comparing the $\mathrm{H} \alpha$ profiles at all four epochs (Fig. 6) shows that the red side of the line changes very little, but there is extra emission that develops with 


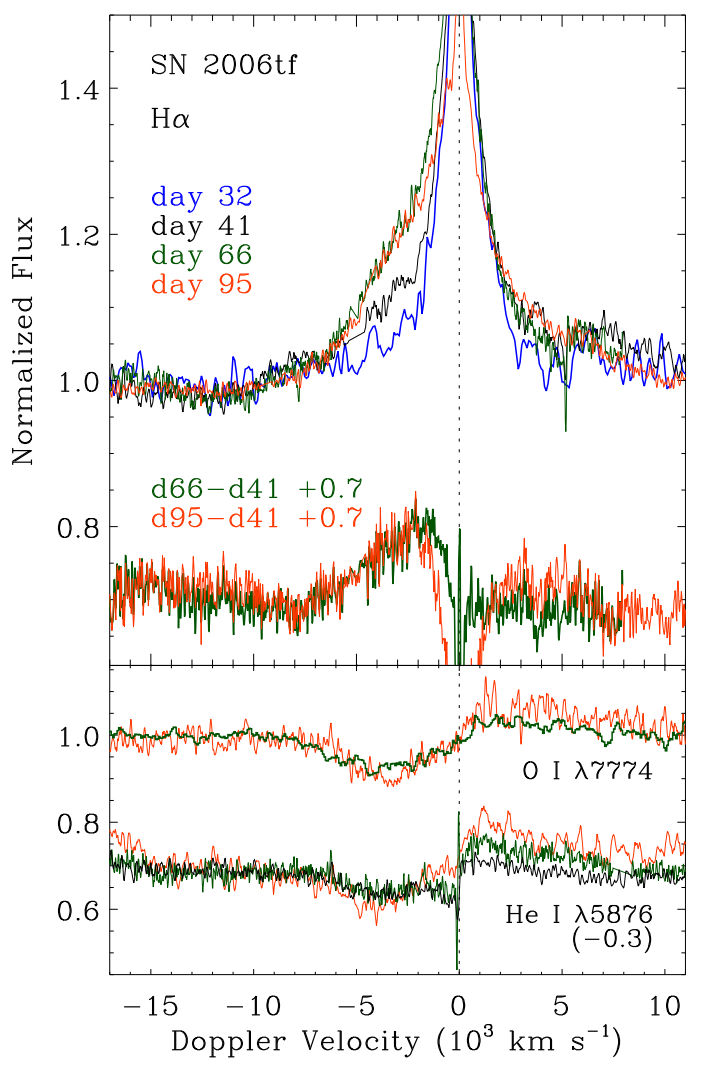

Fig. 8.- (Top panel) Same as Figure 6 but with an expanded velocity scale and smaller vertical range to show the behavior of the broad blueshifted wings of $\mathrm{H} \alpha$. The lower tracings show the day 66 and 95 residual profiles after subtracting off the symmetric day 41 profile. Residual fast blueshifted emission is seen out to roughly $-7500 \mathrm{~km} \mathrm{~s}^{-1}$. (Bottom panel) Line profiles of O I $\lambda 7774$ on day 64 (green) and 95 (orange), and the feature at $\sim 5900 \AA$ (colors for different epochs are the same as the top panel), which could potentially be either He I $\lambda 5876$ or $\mathrm{Na}$ I $\lambda 5892$. It is plotted here as if it were He I $\lambda 5876$ (we have subtracted 0.3 from the normalized flux for display purposes), because that is the correct identification for its narrow P Cygni component.

increasing strength on the broad blueshifted wing. One would normally attribute the apparent flux deficit in the red wing to high optical depth that blocks the far side of the SN, but this hypothesis predicts a trend opposite to the one seen; i.e., if the asymmetry were due to high optical depths alone, then one would normally expect the broad emission to become more symmetric with time.

Figure 8 investigates the blueshifted wings of broadline profiles in more detail. The profile of this "extra" emission on days 66 and 95 is illustrated in Figure 8 by subtracting the symmetric day 41 profile from the latter two epochs. The residual emission shows a broad component to $\mathrm{H} \alpha$ at blueshifted velocities, with its fastest speeds reaching about $-7500 \mathrm{~km} \mathrm{~s}^{-1}$, and little or no corresponding emission on the red side of the line.

Two possible explanations for this fast blueshifted emission are that it is due to electron scattering or to rapidly moving material. If it were due primarily to electron scattering, though, we should see a component of comparable strength on the red side of the line as well.

In fact, the explanation for this fast blueshifted $\mathrm{H} \alpha$ emission is not likely to be electron scattering alone, because we also see evidence for fast-moving blueshifted material in absorption in some other species. The bot- tom panel of Figure 8 shows broad profiles of O I $\lambda 7774$ and He I $\lambda 5876,{ }^{6}$ both of which indicate weak absorption features with ill-defined blue absorption edges out to about -7000 or $-8000 \mathrm{~km} \mathrm{~s}^{-1}$, matching the speeds at which we see enhanced blueshifted $\mathrm{H} \alpha$ emission. We also note that the relative strength of the broad emission component of He I $\lambda 5876$ increases with time.

Since the speeds up to about $7500 \mathrm{~km} \mathrm{~s}^{-1}$ are well in excess of the blast-wave speed we derived in the previous section, the broad emission probably arises in gas that has not yet reached the reverse shock of the CSM interaction region. This expanding material just interior to the reverse shock may be ionized by the backwardpropagating radiation from the post-shock gas (Chevalier \& Fransson 1994). The severe asymmetry of the line means that we are only seeing the near side of the ejecta because of dust formation, high optical depths in the ejecta, or global asymmetry.

The fact that these broad features are also seen in blueshifted absorption in He I $\lambda 5876$ and O I $\lambda 7774$ provides a critical clue to their origin and to the physical processes at work in this SN. To be moving this fast, the material must have not yet reached the reverse shock, but to be seen in absorption, it must also have a background light source. Thus, even as late as day 95, some central source (i.e., the inner SN ejecta?) still contributes a non-negligible fraction of the continuum luminosity.

What constitutes a "non-negligible" fraction in this case? The background continuum source must contribute at least $10 \%$ of the luminosity at red wavelengths, because that is roughly the depth of the absorption in $\mathrm{O}$ I $\lambda 7774$. This requires the source in question to have a luminosity of roughly $8 \times 10^{8} \mathrm{~L}_{\odot}\left(\right.$ or $M_{R} \approx-17.4 \mathrm{mag}$ ) on day 95, which is about 1 mag more luminous than a normal SN II-P at that same time and even somewhat more luminous than a "hypernova" like SN 1998bw at that epoch (Galama et al. 1998). Thus, we must speculate that either the underlying SN ejecta of SN 2006tf were unusually luminous independent of its strong CSM interaction, or that perhaps the strong CSM interaction has somehow rejuvenated the underlying ejecta with inwardpropagating radiation. The alternative is that we would need the $7500 \mathrm{~km} \mathrm{~s}^{-1}$ to get out ahead of the $2000 \mathrm{~km}$ $\mathrm{s}^{-1}$ shell, which seems unlikely without radical departures from spherical symmetry.

In these broad features, we have another clue that SN 2006tf is a more luminous analog of SN 1988Z, which also showed underlying broad components of $\mathrm{H} \alpha$ in the SN ejecta (Stathakis \& Sadler 1991; Turatto et al. 1993). In the case of SN 1988Z, the underlying broad $\mathrm{H} \alpha$ component was also asymmetric, although not as severely as that of SN 2006tf, and it also increased in prominence with time (up to about day 115) and then faded again. In principle, though, the underlying SN ejecta would be easier to see in SN 1988 Z because the total SN luminosity was lower than in SN 2006tf.

\subsection{Narrow $H \alpha$ and $H \beta$ Emission Profiles}

Our spectra at the four epochs are obtained with different spectral resolution (see Table 2), and this may be the

${ }^{6}$ We have labeled this feature as He I $\lambda 5876$ because that places its narrow P Cygni feature at the correct velocity, but the underlying broad feature could also plausibly be identified as $\mathrm{Na}$ I $\lambda 5895$. 


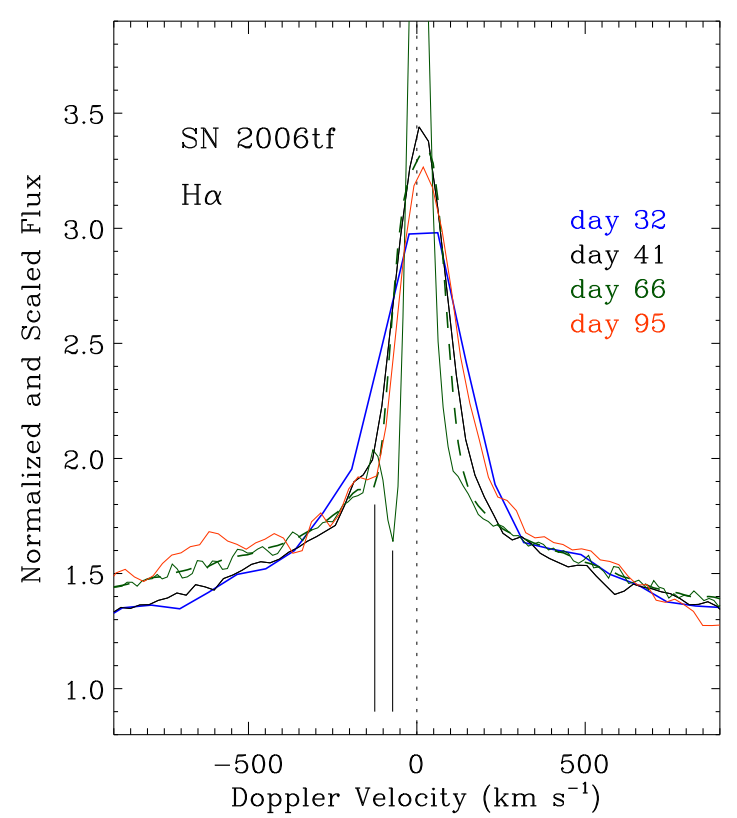

FIG. 9.- Same as Figure 6 but highlighting the narrow $\mathrm{H} \alpha$ component. The scale has been adjusted so that the broad wings of $\mathrm{H} \alpha$ overlap. The day 66 spectrum (green) was obtained with DEIMOS using a higher resolution than the other spectra; it is shown at the observed resolution (solid green), as well as smoothed (dashed green) to match the resolution on days 41 (black) and 95 (orange). At degraded resolution, the $\mathrm{H} \alpha$ profile on day 66 is nearly identical to that on days 41 and 95, suggesting little or no change in the strength of the narrow component or its narrow $\mathrm{P}$ Cygni absorption. The day 32 spectrum (blue) was obtained with even lower resolution. The two vertical dashes mark the velocity of the $\mathrm{P}$ Cygni trough at $-72 \mathrm{~km} \mathrm{~s}^{-1}$ and its blue edge at $-125 \mathrm{~km} \mathrm{~s}^{-1}$.

primary reason for apparent differences in the narrow $\mathrm{H} \alpha$ profile (Fig. 9). ${ }^{7}$ Only one epoch (day 66) had sufficiently high dispersion to fully resolve the line profile. It shows a strong narrow emission component with a width of $\sim 150$ $\mathrm{km} \mathrm{s}^{-1}$ in both $\mathrm{H} \alpha$ and $\mathrm{H} \beta$ at the point where it meets the underlying broader base. This is an underestimate of the emission line's intrinsic full width near zero intensity (FWZI) because a narrow P Cygni absorption feature is also seen in both lines, which partially absorbs the blue side of the line. Blueshifted absorption is stronger in $\mathrm{H} \beta$ than in $\mathrm{H} \alpha$, which was true for the broad components as well (Fig. 5).

The pre-shock CSM speed is best seen in the blueshifted $\mathrm{P}$ Cygni absorption feature of $\mathrm{H} \beta$ on day 66 (Fig. 10), which has a blue edge indicating a progenitor wind speed of $190 \mathrm{~km} \mathrm{~s}^{-1}$. This is, again, similar to the case of SN 2006gy, where the blue edge of the narrow P Cygni absorption indicated a wind speed of roughly $230 \mathrm{~km} \mathrm{~s}^{-1}$ (Smith et al. 2007). As noted in that paper, such wind speeds are much too fast for red supergiants and much too slow for Wolf-Rayet (WR) stars, but they are typical of blue supergiants and especially LBVs. As with SN 2006gy, this is likely to be a critical clue to the nature of the star that exploded and its immediate preSN mass loss that gave rise to its dense CSM.

\footnotetext{
7 However, we do not believe that the lower spectral resolution on day 64 fully accounts for the weaker narrow absorption components of Fe II and other species, as compared to SNe 1994W and 2006 gy in Figure 2 because the spectrum with much higher resolution obtained 2 days later does not show strong narrow absorptin features either.
}

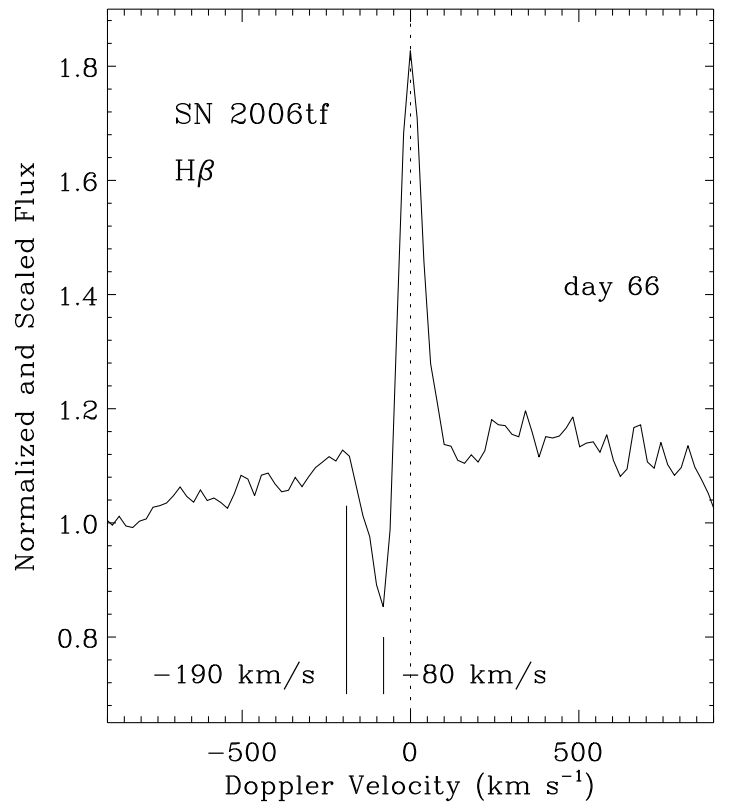

FIG. 10.- Narrow component of the $\mathrm{H} \beta$ profile on day 66 from the high-resolution DEIMOS spectrum. This gives the best estimate of the progenitor's pre-shock wind speed of $190 \mathrm{~km} \mathrm{~s}^{-1}$ from the blue edge of the P Cygni absorption component. The minimum of the blueshifted absorption is at $-80 \mathrm{~km} \mathrm{~s}^{-1}$.

Despite the differences in spectral resolution, we find no clear evidence that the narrow $\mathrm{H} \alpha$ profile is changing with time. When we degrade the spectral resolution of the day 66 spectrum (the dashed green profile in Fig. 9) to match the resolution on days 41 and 95, the narrow $\mathrm{H} \alpha$ emission component has the same profile shape and the $\mathrm{P}$ Cygni absorption feature is no longer seen. This suggests that the same narrow P Cygni absorption may actually be present on days 41 and 95 as well, but is not resolved in those spectra. The first epoch on day 32 has even lower spectral resolution, so a similar comparison is less clear. There is some suggestion of persistent narrow $\mathrm{P}$ Cygni absorption in $\mathrm{H} \beta$ at all epochs (Fig. 5 ).

The strength of the narrow $\mathrm{H} \alpha$ emission component relative to the underlying broad component changes slowly but steadily. If we measure the strength of the narrow emission compared to the total line flux including the broader emission (listed as "N/T" in Table 2), we find that the narrow emission contributes roughly $17 \%$, $11 \%, 8 \%$, and then $3 \%$ of the total $\mathrm{H} \alpha$ emission on days $32,41,66$, and 95, respectively (Table 2). As late as day 445 , this fraction remains at about $3-4 \%$ (see below). Recognition that the narrow component makes a relatively minor contribution to the total line luminosity will be important in $\S 5.5$, where we discuss the behavior of the integrated line luminosities and equivalent widths.

\subsection{Late-Time Ho Emission}

Our late-time spectrum taken on day 445 is shown in Figure 11. This is the first epoch at which we see evidence that the overall character of the spectrum has changed significantly. The continuum emission is very weak, which is the reason for the very large equivalent width in Table 2. Besides $\mathrm{H} \alpha$, faint emission from other Balmer lines and the Ca II near-infrared triplet at $8500 \AA$ can also be seen. The $\mathrm{H} \alpha / \mathrm{H} \beta$ flux ratio has now climbed to $\gtrsim 11.6$, indicating that it is dominated by collisional 


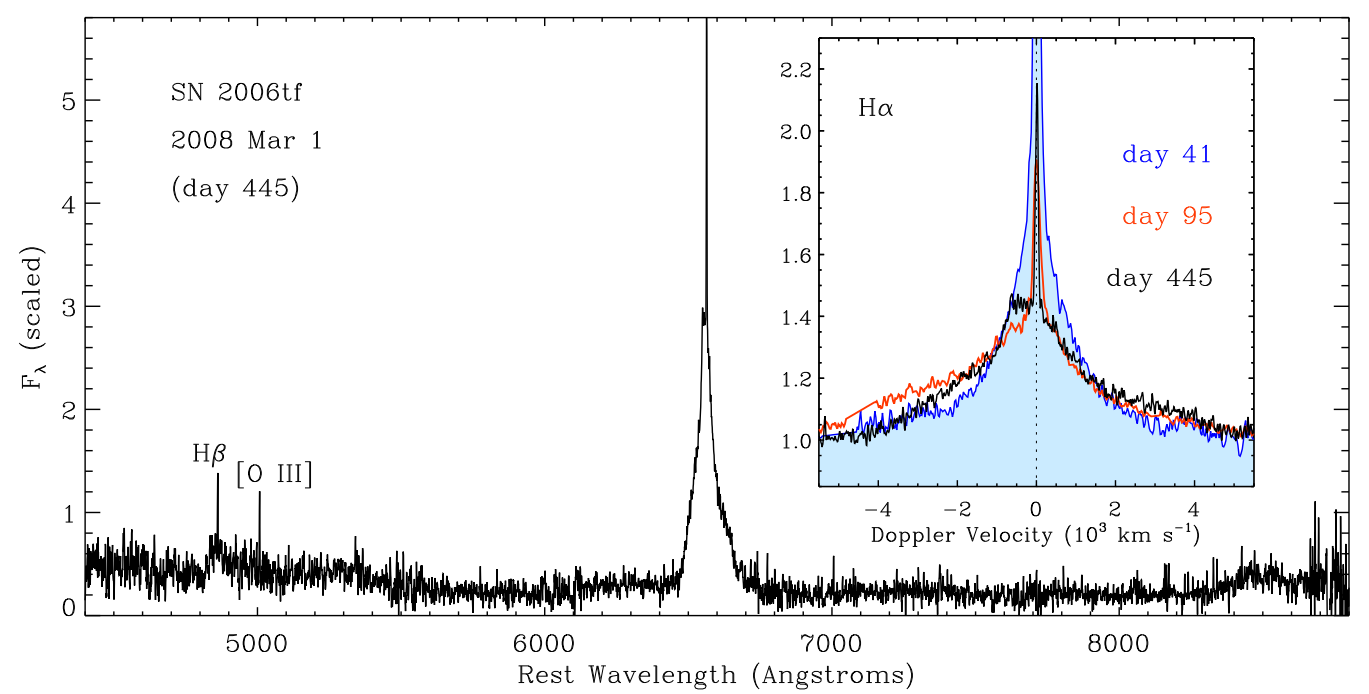

FIG. 11. - The late-time spectrum of SN 2006tf, obtained on 2008 Mar. 1, day 445 after discovery. The inset shows the H $\alpha$ profile on day 445 , scaled arbitrarily and compared to the day 41 (shaded blue) and day 95 (orange) spectra from Figure 6 ] Narrow $\mathrm{H} \beta$ and [O III] $\lambda 5007$ are real and may signify an underlying H II region, but there are many noise spikes redward of the broad near-infrared Ca II emission.

excitation rather than recombination (Raymond et al. 2007).

The broad components out to $7500 \mathrm{~km} \mathrm{~s}^{-1}$ (Fig. 8) are no longer seen. This suggests that the material responsible for these features had reached the reverse shock by day 445. The absence of absorption, in particular, indicates that the power source of their underlying continuum had faded by this time as well, as expected if that continuum source was the underlying SN ejecta.

The spectrum is dominated by a strong $\mathrm{H} \alpha$ emission line with a similar intermediate width as before, as well as a lingering unresolved narrow component that makes up a comparable fraction (about 3-4\%) of the total emission as in the day 95 spectrum. The narrow component is about a factor of 2 narrower than before, though, with a FWHM of $\sim 80 \mathrm{~km} \mathrm{~s}^{-1}$, and reaching $\pm 80 \mathrm{~km} \mathrm{~s}^{-1}$ at its base. The narrow $\mathrm{P}$ Cygni absorption component is no longer present, even though the late-time spectrum has higher resolution than our day 66 spectrum. The luminosity of the narrow component is a factor of $\sim 6$ fainter than on day 95, with a luminosity of only $2.5 \times$ $10^{39} \mathrm{ergs} \mathrm{s}^{-1}$. This very narrow $\mathrm{H} \alpha$ emission is likely to be at least partially contaminated by a background H II region, since narrow components of $\mathrm{H} \beta$ and [O III] $\lambda 5007$ are also seen (Fig. 11).

The inset of Figure 11 shows a detail of the $\mathrm{H} \alpha$ line, compared to the early-time profiles on days 41 and 95. The broader component of the line has a similar width as before, and shows complex behavior. At some velocities, the line wings match the symmetric day 41 profile, while at other velocities the late-time line wings are similar to those on day 95. These are probably important clues to the optical depth and geometry as the SN evolves.

One very interesting quality of the late-time $\mathrm{H} \alpha$ profile is the pronounced asymmetry at relatively low velocities of $\pm 1000 \mathrm{~km} \mathrm{~s}^{-1}$. Within this range, the intermediatewidth component is very asymmetric and blueshifted as compared to the symmetric day 41 profile, with a sharp edge on the blue side where it meets the line wing.

An intriguing possibility is that the net blueshift of the line may be due to dust formation in the dense post-shock cooling shell, analogous to the well-established case of the peculiar SN Ib 2006jc (Smith et al. 2008a), and possibly also the SNe IIn 2005ip (R. Chevalier 2008; priv. comm.) and 1998S (Pozzo et al. 2004). The optical depth would be highest at the limbs of that shell, favoring the extinction of material at low redshifted velocities and speeds near zero. If dust has formed in the post-shock region of SN 2006tf, we would predict excess emission in nearinfrared bands, analogous to the case of SN 2006jc, and possibly also signs of increased extinction. In that case, the fact that dust formed much later in SN 2006tf (days 200-400 instead of by day 50 in SN 2006jc) can be understood as a consequence of the fact that SN 2006jc faded much more rapidly than SN 2006tf, and was much less luminous overall. Dust formation could also mean that the late-time continuum we measure is an underestimate.

\subsection{Line Luminosities and Equivalent Widths}

Figures 12 and 13 show how the strength of Balmer emission changes with time. We measured the equivalent widths of the total $\mathrm{H} \alpha$ and $\mathrm{H} \beta$ emission at the epochs for which we have spectra, and used the red continuum flux inferred from the light curve to derive the total $\mathrm{H} \alpha$ line flux. ${ }^{8}$ We also measured the $\mathrm{H} \alpha / \mathrm{H} \beta$ flux ratio and the fractional contribution of the narrow CSM emission components to the total $\mathrm{H} \alpha$ flux in our spectra. These are listed in Table 2. Uncertainties in the $\mathrm{H} \alpha$ flux are typically $5-8 \%$, depending on the noise in the continuum.

The luminosity of the narrow component declines slowly, roughly following the same decline rate as the continuum luminosity inferred from the light curve (Fig. 12 shows the same diffusion model curve from Fig. 4 divided by a factor of 2900 for comparison). Thus, the narrow $\mathrm{H} \alpha$ emission represents a constant $0.034 \%$ of the bolometric luminosity, and its effective equivalent width would be constant if it were measured relative to the underlying continuum. This implies that the narrow emission from the pre-shock CSM is radiatively excited by the same continuum source that powers the bolometric lu-

\footnotetext{
${ }^{8}$ However, the $\mathrm{H} \alpha$ luminosity on day 445 was measured directly from the flux-calibrated spectrum, although that flux was uncertain by roughly $50 \%$ because of possible light cirrus clouds.
} 


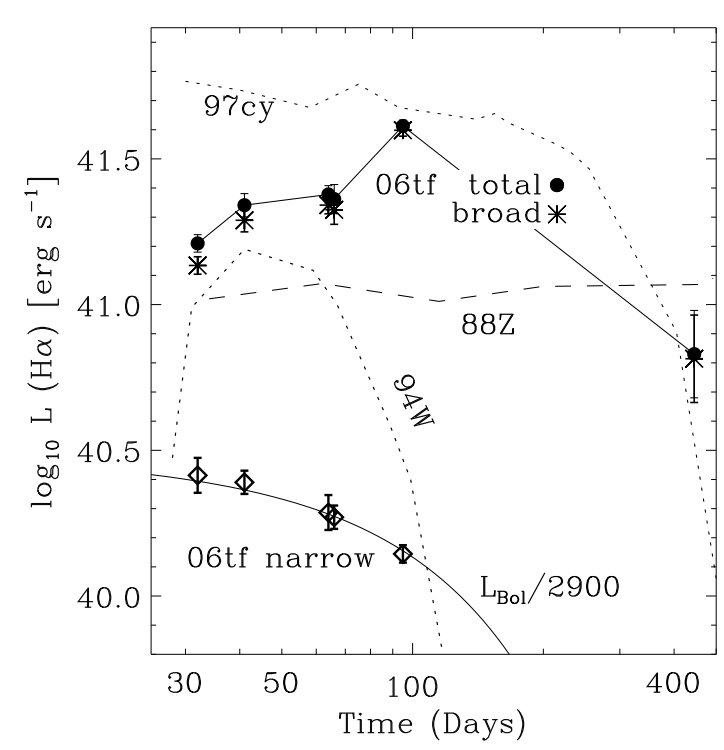

FIG. 12.- The intrinsic luminosity of the $\mathrm{H} \alpha$ emission line in SN 2006tf, showing the total flux (solid dots), the broad/intermediate-width component from the post-shock gas (asterisks), and the narrow component from the unshocked CSM (diamonds), from Table 2. The decline rate of the narrow component of $\mathrm{H} \alpha$ in SN 2006tf follows the decline rate of the continuum luminosity (the model diffusion curve from Fig. 4 divided by 2900; solid curve). For comparison, we also show the total $\mathrm{H} \alpha$ luminosities of SN 1994W (dotted line) from Chugai et al. (2004), and SN 1988Z (dashed line) from Stathakis \& Sadler (1991) and Turatto et al. (1993), as well as SN 1997cy. [These SN 1997cy measurements were taken from Fig. 11 of Pastorello et al. (2002). A. Pastorello (2008, private comm.) informed us that they were made from spectra presented by Turatto et al. (2000).]

minosity, but not the same source that powers the broad post-shock $\mathrm{H} \alpha$ emission. If this is Case $\mathrm{B}$ recombination emission, the mass of $\mathrm{H}$ gas needed to create the $\mathrm{H} \alpha$ line can be expressed as $M_{\mathrm{H} \alpha}=11.4 \mathrm{M}_{\odot}\left(L_{\mathrm{H} \alpha} / n_{e}\right)$, where $L_{\mathrm{H} \alpha}$ is expressed in $\mathrm{L}_{\odot}$ and $n_{e}$ is the characteristic electron density in the CSM in $\mathrm{cm}^{-3}$. For the narrow $\mathrm{H} \alpha$ component in SN 2006tf, the value of $L_{\mathrm{H} \alpha} \approx 5 \times 10^{6}$ $\mathrm{L}_{\odot}$ at early times implies a very large mass of pre-shock CSM - even for very high CSM densities, the total mass required is $\sim 6 \mathrm{M}_{\odot}\left(n_{e} / 10^{7} \mathrm{~cm}^{-3}\right)^{-1}$. If this emitting material resides within a radius of $\sim 10^{16} \mathrm{~cm}$, then the average density is $\sim 10^{8} \mathrm{~cm}^{-3}$, implying that the progenitor's wind had a mass-loss rate of $\sim 0.1 \mathrm{M}_{\odot} \mathrm{yr}^{-1}$ for many decades before the SN, and prior to the even more extreme mass loss in the few years before explosion.

The broad/intermediate-width component (and hence, the total $\mathrm{H} \alpha$ line luminosity) exhibits very different behavior from that seen in the narrow CSM lines (Fig. 12). Instead of fading with the continuum luminosity, the $\mathrm{H} \alpha$ luminosity of the post-shock gas rises dramatically as the SN fades. An important clue to this behavior is that the nature of the intermediate-width Balmer emission changes dramatically during this time (see Table 2 ): at early times, the $\mathrm{H} \alpha / \mathrm{H} \beta$ ratio matches the value one expects for recombination emission, suggesting that the heating of the post-shock gas emitting $\mathrm{H} \alpha$ is actually dominated by photoionization heating. This changes with time, however, as the ratio climbs to $\mathrm{H} \alpha / \mathrm{H} \beta>10$, indicative of pure collisional excitation. It can also be seen in the equivalent-width behavior (Fig. 13), where

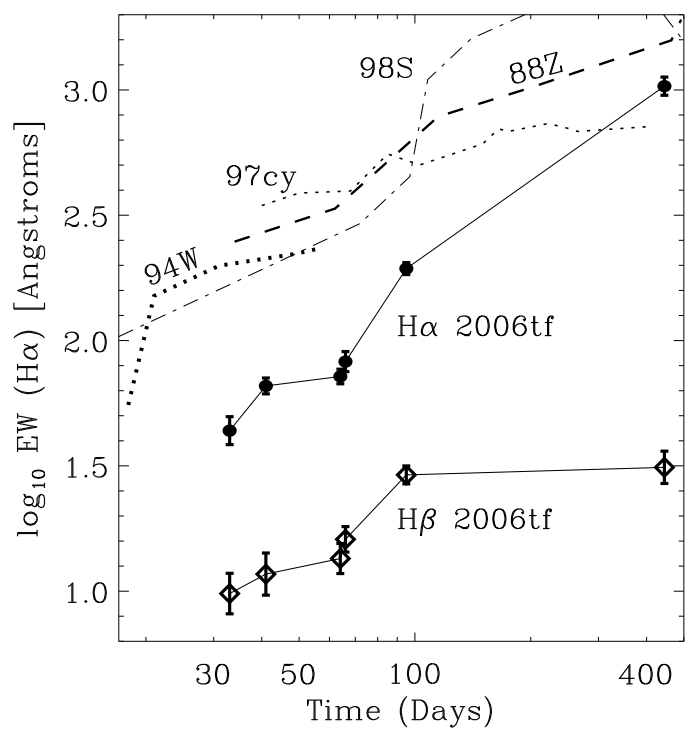

FIG. 13.- The equivalent widths of $\mathrm{H} \alpha$ and $\mathrm{H} \beta$ emission with time, measured in our spectra of SN 2006tf (emission-line equivalent widths are positive and include narrow, broad, and intermediate-width components). Evolution of the $\mathrm{H} \alpha$ equivalent width is shown for several other representative SNe IIn discussed in the text. Except for SN 1998S (Leonard et al. 2000), most objects do not have published $\mathrm{H} \alpha$ equivalent widths, so we derived them from $R$-band photometry and published $\mathrm{H} \alpha$ line fluxes from several sources cited in the text.

$\mathrm{H} \alpha$ continues to rise, while $\mathrm{H} \beta$ levels off.

This suggests that as time proceeds, SN 2006tf transitions from an optically thick regime where radiative heating dominates the emission, to a regime where collisional heating by ongoing CSM interaction dominates. In some sense, this will be true for any strongly interacting SN IIn, because the highest optical depths are usually encountered at early times. One clear manifestation of such behavior is an increasing line-to-continuum ratio, measured as the equivalent width. Figure 13 compares the $\mathrm{H} \alpha$ equivalent width of SN 2006tf to that of several other SNe. All SNe IIn shown here have $\mathrm{H} \alpha$ equivalent widths that increase with time as the densities drop and the underlying continuum fades. However, it is clear that even though the $\mathrm{H} \alpha$ luminosity of SN 2006tf is not too different from some of these other SNe (Fig. 12), its $\mathrm{H} \alpha$ equivalent width is systematically less than all others during the main peak of its light curve, and it does not catch up until late times (Fig. 13). This discrepancy would be even more stark if we accounted for the likely fact that the explosion date should be at least $30 \mathrm{~d}$ before the discovery date, shifting the SN 2006tf data to the right in Figure 13. This is a sign that the continuum luminosity contributed by photon diffusion from optically thick material is much stronger in SN 2006tf than in the fainter SNe IIn, and that this component persists much longer. At early times, the fraction of the bolometric luminosity contributed by the $\mathrm{H} \alpha$ line, $L_{\mathrm{H} \alpha} / L_{\mathrm{Bol}}$, is about $0.4 \%$, rising to about $2.6 \%$ at late times. Corresponding numbers for the other SNe IIn in Figure 13 are $L_{\mathrm{H} \alpha} / L_{c} \approx 2 \%$ at early times and about the same as the of SN 2006tf at late times.

The different behavior with time in SN 2006tf suggests that it would be difficult to fully account for its high 


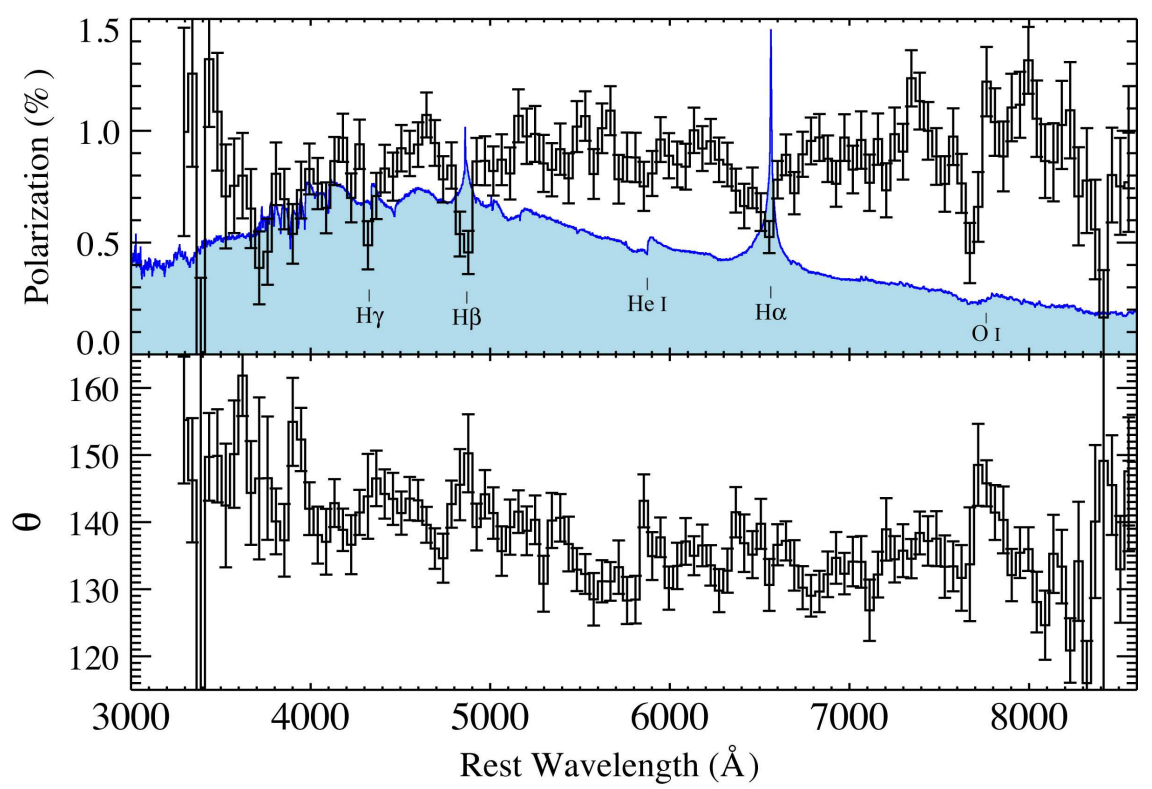

FIG. 14. - Spectropolarimetry of SN 2006tf on day 64. The top panel shows the observed polarization (estimated using a rotated Stokes parameter) in black with error bars, binned to 50 observed $\AA$ pixel ${ }^{-1}$ for clarity. Overplotted in blue (shaded gray in the printed edition) is the total-flux spectrum from that date for reference. The continuum polarization between the lines is about $0.9 \%$, with decreases in polarization visible at the wavelengths of the Balmer emission lines and especially O I $\lambda 7774$. The bottom panel shows the observed position angle of the polarization.

luminosity with a model like the one applied to most other SNe IIn, where $\mathrm{H} \alpha$ and continuum emission cool the post-shock gas in quasi-steady-state. The difference in the case of SN 2006tf is that the extra continuum luminosity may be due to delayed photon diffusion from an opaque shocked shell, as discussed earlier. Without a two-component model like this, it is difficult to see why the continuum luminosity of SN 2006tf would be a factor of 4-10 higher than that of SNe $1994 \mathrm{~W}$ and $1988 \mathrm{Z}$ even though its $\mathrm{H} \alpha$ luminosity is only 1.5-2 times larger.

Eventually, as the radius increases and the density drops, SN 2006tf becomes dominated by direct radiation from ongoing optically thin CSM interaction, very much like the late-time behavior of SN $1988 \mathrm{Z}$ and other SNe IIn. However, the drop in the total $\mathrm{H} \alpha$ luminosity at late times suggests that the progenitor mass-loss rate of SN 2006tf was not constant, but began to rise as the time of core collapse approached (see $\S 7$ ).

\section{SPECTROPOLARIMETRY}

The spectropolarimetry of SN 2006tf on day 64 is plotted in Figure 14. The continuum is polarized at about the $0.9 \%$ level, with several depressions in the polarization at the locations of the strong Balmer emission lines and $\mathrm{O}$ I $\lambda 7774$. For $\mathrm{H} \alpha$ and $\mathrm{O}$ I especially, the depolarization seems to be primarily associated with the broad blueshifted components discussed in $\$ 5.2$ and Figure 8 . The differing polarization of the lines and continuum indicates that at least some of the observed polarization must be intrinsic to the supernova and not simply due to ISP. We integrated the observed polarization over the spectral range 5050-5950 $\AA$ in the rest frame of the supernova to simulate a rest-frame $V$-band observation and obtained a value of $P_{V}=0.91 \pm 0.03 \%$ for the continuum polarization at a position angle of $\theta=135^{\circ} .4 \pm 0.8$.

The few SNe IIn studied polarimetrically in the past have hinted that the objects as a class exhibit high polar- izations and hence large asymmetries. The first evidence that this might be true came from broad-band imaging polarimetric observations of SN $1994 \mathrm{Y}$ about 245 d after discovery (Wang et al. 1996). The $R$-band polarization for that object (dominated by $\mathrm{H} \alpha$ ) differed by more than $1.5 \%$ (defined as the difference in the Stokes parameters $q$ and $u$ added in quadrature) from that measured in the $B$ and $V$ bands, which were dominated by continuum emission. Wang et al. (1996) concluded that SN 1994Y had significant intrinsic polarization, but without spectropolarimetry the interpretation was unclear.

Subsequently, spectropolarimetric sequences of two SNe IIn have appeared in the literature. SN 1998S was observed within a week of explosion (Leonard et al. 2000) and twice at later epochs (Wang et al. 2001). Hoffman et al. (2008) recently presented three epochs of spectropolarimetry of SN 1997eg ranging from 16 to 93 d after discovery. The initial continuum polarization was observed to be large in both objects $(P \approx 2.0 \%$ in SN $1998 \mathrm{~S}$ and $P \approx 2.3 \%$ in SN 1997eg), with modulations larger than $1.5 \%$ across the strongest emission lines. Both objects also showed time-variable polarization in the continuum and lines, with the polarization changing by more than a percent in the $q-u$ plane.

Despite the high data quality for these two SNe, interpretation has been complicated. Observed continuum polarization can be attributed to both ISP by dust along the line of sight and intrinsic continuum polarization of the SN due to electron scattering. Scattering by lines is believed to be intrinsically depolarizing, but if the linescattering material is not distributed spherically, it may produce net polarization when the spatially unresolved $\mathrm{SN}$ is observed from afar. Finally, as discussed above, emission-line profiles show several components representing material with potentially different spatial distributions. The earliest epoch of data on SN 1998S (Leonard et al. 2000) showed separate polarization modulations in 
TABLE 3

Some Basic Physical Properties of SN 2006 $\mathrm{TF}^{\mathrm{a}}$ in a CSM Interaction Scenario

\begin{tabular}{lccccccrc}
\hline \hline Day & $\begin{array}{c}\mathrm{L}_{\text {Bol }} \\
\left(10^{9} \mathrm{~L}_{\odot}\right)\end{array}$ & $\begin{array}{c}\mathrm{T}_{B B} \\
(\mathrm{~K})\end{array}$ & $\begin{array}{c}R_{B B} \\
\left(10^{15} \mathrm{~cm}\right)\end{array}$ & $\begin{array}{c}R_{\text {shell }} \\
\left(10^{15} \mathrm{~cm}\right)\end{array}$ & $\zeta$ & $\begin{array}{c}w \\
\left(10^{18} \mathrm{~g} \mathrm{~cm}^{-1}\right)\end{array}$ & $\begin{array}{c}\dot{M}_{\mathrm{CSM}} \\
\left(\mathrm{M}_{\odot} \mathrm{yr}^{-1}\right)\end{array}$ & $\begin{array}{c}t_{\dot{M}} \\
(\mathrm{yr})\end{array}$ \\
\hline 32 & 13.6 & 7800 & 4.52 & 4.56 & 0.98 & 13.6 & 4.1 & -7.6 \\
41 & 12.7 & 7500 & 4.72 & 4.72 & 1.00 & 12.7 & 3.8 & -7.9 \\
64 & 10.5 & 6800 & 5.22 & 5.12 & 1.04 & 10.5 & 3.1 & -8.6 \\
66 & 10.3 & 6800 & 5.18 & 5.16 & 1.02 & 10.3 & 3.1 & -8.6 \\
95 & 7.9 & 6300 & 5.26 & 5.66 & 0.86 & 7.9 & 2.3 & -9.5 \\
445 & 0.64 & {$[6300]$} & {$[1.5]$} & 11.7 & {$[0.016]$} & 0.64 & 0.2 & -20 \\
& & & & & & & &
\end{tabular}

a $R_{\text {shell }}$ and properties in columns to its right are derived assuming a constant shell expansion speed of $2000 \mathrm{~km} \mathrm{~s}^{-1}$. Highly uncertain values are in square brackets

both narrow and broad lines, prohibiting any interpretation that allowed both components to be completely depolarizing. Hoffman et al. (2008) found that the polarization of strong lines $(\mathrm{H} \alpha, \mathrm{H} \beta$, and $\mathrm{He}$ I $\lambda 5876)$ in SN 1997eg showed "loops" when plotted in the Stokes $q-u$ plane. They attributed this to the differing geometries of narrow- and broad-line material, and thus to a mismatch in symmetry axes of SN ejecta and the CSM.

Our observations of SN 2006tf do not have sufficient signal-to-noise ratio to disentangle all these effects, especially from a single epoch of data, but we can make useful comparisons to SNe 1998S and 1997eg. The first point to note is that the observed continuum polarization of SN 2006tf is lower than that of the other two objects, both of which showed polarizations of at least $2 \%$ at early times. However, both objects showed significant ISP, making any estimate of the intrinsic continuum polarization difficult and model dependent. Also, the continuum polarization changed over time, with that of SN 1997eg declining to $\sim 1.5 \%$ by day 93 . Our SN $2006 \mathrm{tf}$ observations were taken at least $64 \mathrm{~d}$ after explosion, so one possibility is that the polarization was higher at earlier times, but the optical depth to electron scattering declined with time, perhaps as a result of recombination or decreasing densities in the ejecta.

Potential evidence that the intrinsic continuum polarization is actually lower in SN 2006tf than in the other two objects is that the depolarization of line features is significantly smaller. As mentioned above, the line features in the other objects were stronger than $1.5 \%$. In SN 2006tf, the modulation at $\mathrm{H} \alpha$ is only about $0.4 \%$. Quantitatively, the Stokes parameters $(q, u)$ integrated over $6555-6575 \AA$ (the core of the narrow $\mathrm{H} \alpha$ line) are $(-0.27,-0.60)$ with an uncertainty of $\pm 0.09 \%$ for each. This value represents a difference of $0.41 \%$ in polarization (Stokes parameters added in quadrature) from the $V$-band value quoted above, corresponding to $\sim 1 / 4$ of the polarization in the other objects. Overall, then, our general conclusion is that SN 2006tf is only moderately polarized at late times, showing no clear sign for extreme large-scale asymmetry in the CSM interaction region.

\section{BASIC PHYSICAL PROPERTIES OF SN 2006tf}

In the preceding sections, we have described the basic energy demands, overall spectral properties, kinematics from line profiles, and line luminosities that are important clues to the nature and evolution of SN 2006tf. With these in hand, we can now quantify some of the fundamental physical properties of the SN, listed in Table 3 for each day since discovery when we have spectra. Column

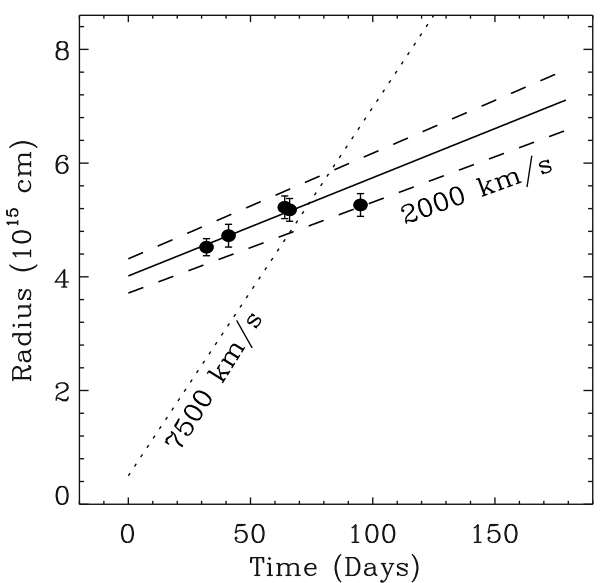

FIG. 15.- Filled dots show the derived black-body radius from Table 3, appropriate for the luminosity and temperature derived from the spectra in Figure 2 The solid line shows the radius for the observed nominal shock speed of $2000 \mathrm{~km} \mathrm{~s}^{-1}$, and the two dashed lines show representative inner and outer radii where the thickness of the post-shock shell is $\sim 15 \%$ of the radius. The dotted line shows radii for an expansion speed of $7500 \mathrm{~km} \mathrm{~s}^{-1}$, where SN ejecta can almost reach the reverse shock by day 64 when the fast blueshifted emission is seen at those speeds.

2 lists the bolometric luminosity (Fig. 4) derived from $R$-band photometry, and column 3 lists the characterstic black-body temperatures for the continuum shapes in Figure 2, These are used to calculate the corresponding radius that a black body would have, listed as $R_{B B}$ in column 4 . In column $5, R_{\text {shell }}$ is the radius of the shell expanding at a constant speed of $2000 \mathrm{~km} \mathrm{~s}^{-1}$, starting from a large initial radius suitable for $R_{B B}$ at the early epochs. These two quantities are also plotted in Figure 15. Column 6 lists the corresponding dilution factor, $\zeta=R_{\mathrm{BB}}^{2} / R_{\text {shell }}^{2}$, which provides an estimate of the effective optical depth or geometric covering factor of the emitting regions of the shell. This dilution factor is a key component in the transition from optically thick to optically thin, as we will discuss later. The next two columns give the wind-density parameter $w=\dot{M}_{\mathrm{CSM}} / V_{\mathrm{CSM}}$ and the progenitor mass-loss rate $\dot{M}_{\mathrm{CSM}}$. These are the values needed to account for the observed bolometric luminosity of SN 2006tf in a simple CSM-interaction model with $100 \%$ efficiency, where the observed luminosity is emitted instantaneously by the post-shock gas, calculated from the expression 


$$
\dot{M}_{\mathrm{CSM}}=2 L \frac{V_{\mathrm{CSM}}}{\left(V_{\mathrm{shell}}\right)^{3}},
$$

where $V_{\mathrm{CSM}}=190 \mathrm{~km} \mathrm{~s}^{-1}$ is the observed pre-shock CSM wind speed, and $V_{\text {shell }}=2000 \mathrm{~km} \mathrm{~s}^{-1}$ is the observed constant speed of the post-shock shell. Again, these provide an estimate of the mass-loss rate that created the CSM being swept up to produce the observed luminosity. Since this assumes $100 \%$ efficiency, it may be an underestimate; any clumping or global asphericity will raise the required values of $w$ and $\dot{M}_{\mathrm{CSM}}$. Accounting for possible broadening of the line wings by electron scattering would raise $\dot{M}_{\mathrm{CSM}}$ even further because it would imply a smaller value of $V_{\text {shell }}$. The last column lists the CSM flow timescale $t_{\dot{M}}=R_{\text {shell }} / V_{\mathrm{CSM}}$, which is the number of years prior to core collapse when the progenitor had the mass-loss rate in the previous column.

The quantities in the last two columns are of particular interest, because they provide a roadmap for what the progenitor star was doing in the decades before it died. This indicates that the progenitor underwent a sudden boost in mass loss in the decade just before exploding. A caveat is that the instantaneous mass-loss rate may not have been quite as high as the peak values around $4 \mathrm{M}_{\odot}$, because diffusion of radiation from an optically thick shocked shell has the effect that the luminosity from earlier CSM interaction, when the material was highly opaque, will effectively "pile up" and mimic a larger instantaneous mass-loss rate. Even allowing for this effect, though, average mass-loss rates of order $2 \mathrm{M}_{\odot}$ $\mathrm{yr}^{-1}$ are needed in the decade before explosion. Before that time (at $t$ minus $20 \mathrm{yr}$ ), the mass-loss rate was apparently 10 times less.

As we have noted elsewhere for SN 2006gy (Smith et al. 2007, 2008b; Smith \& McCray 2007), the only stars known to be capable of producing such extreme mass-loss rates of this order are LBVs during their giant eruptions (Smith \& Owocki 2006), like the 19th-century eruption of $\eta$ Carinae when the star ejected $12.5 \mathrm{M}_{\odot}$ in about a decade (Smith et al. 2003). Even the factor of 10 lower mass-loss rate of $0.1-0.2 \mathrm{M}_{\odot} \mathrm{yr}^{-1}$ needed in the decades before that for SN 2006tf is still 1000 times stronger than the maximum rate that can be supplied by a line-driven wind of a massive star (Smith \& Owocki 2006). However, 0.1-0.2 $\mathrm{M}_{\odot} \mathrm{yr}^{-1}$ is comparable to the less extreme LBV eruption of P Cygni in 1600 AD (Smith \& Hartigan 2006). Gal-Yam et al. (2007) have also discussed LBVs as potential SN IIn progenitors for independent reasons. If the stars that exploded as $\mathrm{SNe} 2006 \mathrm{tf}$ and 2006 gy were not actually LBVs, then they have done a remarkably good job of impersonating the H-rich composition, amount of mass ejected, and ejection speeds of giant LBV eruptions.

\section{SUMMARY AND DISCUSSION}

\subsection{Summary of Observational Clues}

The dataset we have presented above includes many different insights to the nature of SN 2006tf that weave a complex picture of a very luminous SN with remarkably strong CSM interaction. Figure 16 shows an illustration of the essential structural elements of SN 2006tf following the hypothesis that it is a case of extremely strong
CSM interaction, including diffusion from an opaque shocked shell, as mentioned many times throughout this paper. We now summarize the key observational clues of SN 2006tf presented in our study, along with the main implications of each and how they might be understood in a simplified model like that portrayed in Figure 16.

1. The total energy radiated at visual wavelengths is at least $7 \times 10^{50}$ ergs. It could be substantially more depending on how soon after explosion SN 2006tf was discovered. If momentum is conserved, then at best, roughly half the initial kinetic energy can be converted into radiation in a CSM-interaction model. This suggests that the expanding shell should retain roughly an equal amount of kinetic energy. Indeed, our estimated shell mass of $18 \mathrm{M}_{\odot}$ expanding at $2000 \mathrm{~km} \mathrm{~s}^{-1}$ does yield $7 \times 10^{50}$ ergs. This requires a total initial explosion energy of at least $1.4 \times 10^{51} \mathrm{ergs}$ (not including the kinetic energy of fast SN ejecta that have not yet reached the reverse shock). In other words, the very large CSM mass we estimate is comparable to that which is needed to absorb momentum and decelerate the blast wave to only 2000 $\mathrm{km} \mathrm{s}^{-1}$

2. The decline rate of the optical light curve roughly matches that expected for the decay rate of ${ }^{56} \mathrm{Co}$, including the late-time photometry more than 1 yr after explosion (Fig. 4). The initial mass of ${ }^{56} \mathrm{Ni}$ needed to provide such a high luminosity is about $4.5 \mathrm{M}_{\odot}$. In that case, a pair-instability explosion would need to be invoked, as in the case of SN 2006gy if it was also powered by radioactive decay (Smith et al. 2007). In our estimation, however, the strong signature of CSM interaction in the spectrum makes the radioactive-decay hypothesis less palatable for SN 2006tf.

3. The decline rate of the optical light curve during its main peak is fit even better by a simple model of photon diffusion from an opaque shocked shell (Smith \& McCray 2007). This model requires a shell mass of roughly $18 \mathrm{M}_{\odot}$ to account for the long diffusion time and high luminosity, which matches the large mass needed to decelerate the blast wave as noted in point (1) above. The initial radius of the opaque shocked shell is about $180 \mathrm{AU}$ or $\sim 3 \times 10^{15} \mathrm{~cm}$, close to the radius one might expect shortly before discovery (Fig. 15).

4. However, both radioactivity and this diffusion model fall short of the luminosity needed to power the late-time photometry of SN 2006tf (Fig. 4). The similar CSM-interaction model by Woosley et al. (2007), requiring roughly $25 \mathrm{M}_{\odot}$ of CSM (but admittedly tailored for SN 2006gy instead of SN 2006tf), has the same difficulty (Fig. (4). Thus, in any CSM-interaction model, we need to invoke additional, ongoing CSM interaction at large radii to explain the late-time behavior. Indeed, such ongoing CSM interaction is evidenced by the strong $\mathrm{H} \alpha$ emission at late times. The progenitor mass-loss rate needed to account for the late-time luminosity through CSM interaction is about $0.2 \mathrm{M}_{\odot} \mathrm{yr}^{-1}$, about $20 \mathrm{yr}$ before explosion. This is within a factor of 2 of the value required by the narrow CSM component (see next point).

5. The narrow emission and P Cygni absorption components of $\mathrm{H} \alpha$ and $\mathrm{H} \beta$ reveal that the pre-shock CSM is expanding at roughly $190 \mathrm{~km} \mathrm{~s}^{-1}$. This is an important clue to the nature of the progenitor star, because it makes a red supergiant or WR-star progenitor seem unlikely, while the speed is comparable to those of LBVs 

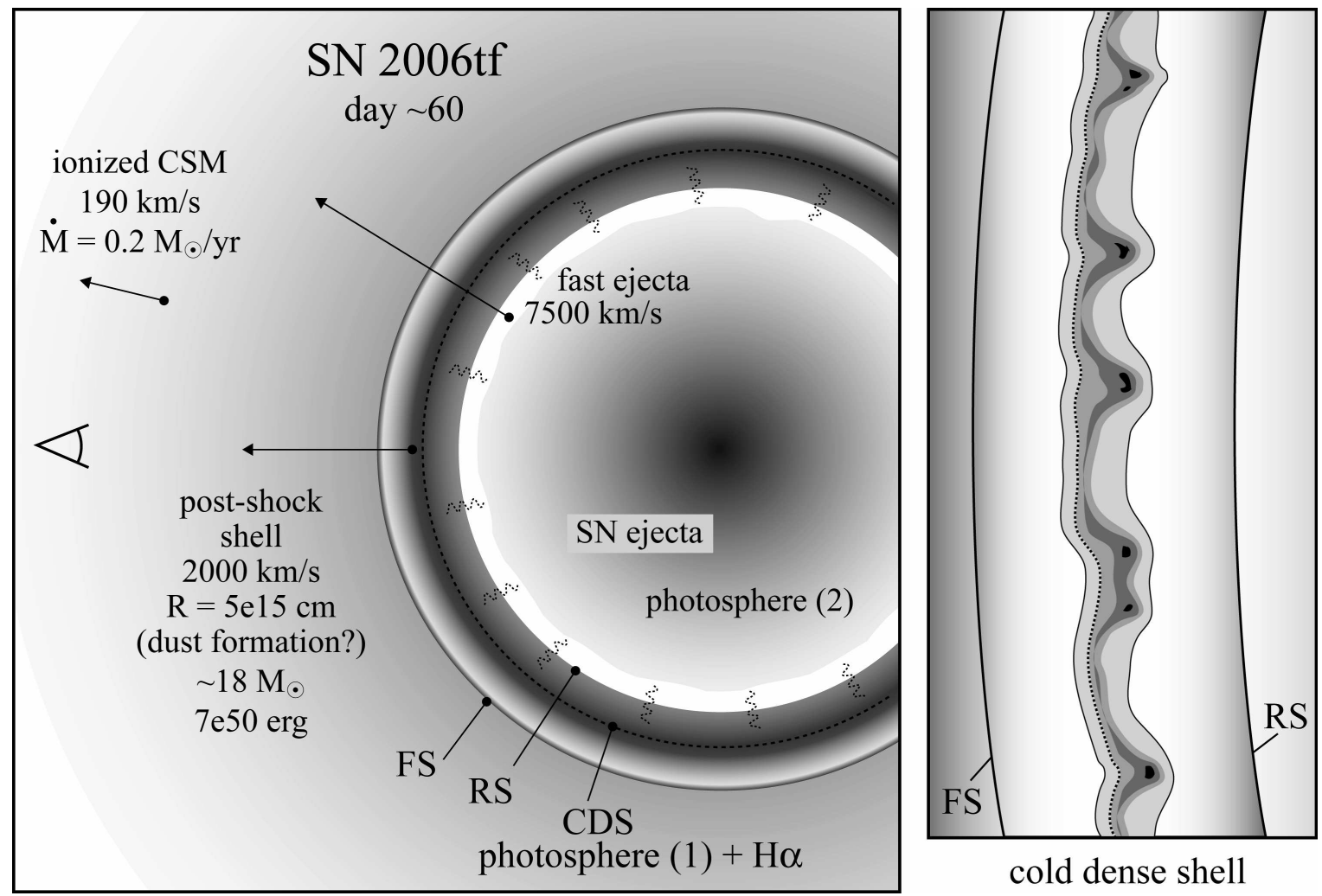

FIG. 16. - Cartoon illustration of the components of SN 2006tf at about $60 \mathrm{~d}$ after discovery, during the decline from the main light-curve peak. The primary feature is the massive post-shock shell of gas, composed of the swept-up opaque pre-SN envelope around the star ejected in the decade before core collapse. Most of the mass is in the cold dense shell (CDS), bounded by the forward shock (FS) and the reverse shock $(\mathrm{RS})$. Diffusion of radiation from this shocked shell produces the main continuum photosphere (1) and the intermediate-width component of $\mathrm{H} \alpha$. This shell expands at constant speed into the pre-shock CSM (dense wind of the progenitor). The interior of the shell is filled by freely expanding SN ejecta, the outermost parts of which are ionized by radiation (wavy lines) propagating inward from the reverse shock, exciting the broad He I and O I features seen in the spectrum. There is also a second photosphere (2) in the SN ejecta, which is fainter than the main photosphere and can only be seen if the main shell thins or develops clumps as time proceeds. The right panel shows a more detailed depiction of the post-shock gas, including the clumpy structure that forms due to instabilities in the cold dense shell layer. The dashed line here represents the photosphere at some arbitrary early time, working its way from left to right through the clumpy CDS as the SN expands. When it reaches a dense clump, the recombination photosphere will proceed through that clump, but for the regions between clumps it will eventually break through, allowing an observer to see the underlying SN ejecta.

and other blue supergiants. The luminosity of the narrow $\mathrm{H} \alpha$ component implies a progenitor mass-loss rate at large radii outside the shock $\left(\sim 10^{16} \mathrm{~cm}\right.$; ejected about 20 yr before core collapse) of at least $0.1 \mathrm{M}_{\odot} \mathrm{yr}^{-1}$. This is close to the value needed to power the late-time luminosity (see previous point), and fully consistent within the uncertainty of the late-time luminosity estimate. This is also a factor of $\sim 10$ lower than the necessary mass-loss rate in the decade just before core collapse, signifying a sharp boost in $\dot{M}$ immediately before the star's death.

6. The intermediate-width component of the $\mathrm{H} \alpha$ line arises mostly in a swept-up, dense, post-shock cooling shell expanding at a constant speed of $\sim 2000 \mathrm{~km} \mathrm{~s}^{-1}$ (Fig. 16). This is the dominant speed of the forward shock plowing into the CSM. This speed does not change perceptibly from day 32 onward. Since the shell does not decelerate even though it is emitting almost $10^{51} \mathrm{ergs}$, the shell must already be very massive by day 32 , consistent with our estimates above.

7. The nature of the Balmer emission changes with time. At early times, the $\mathrm{H} \alpha / \mathrm{H} \beta$ flux ratio is consistent with recombination, whereas at late times, the $\mathrm{H} \alpha / \mathrm{H} \beta$ ratio rises to more than 10 , suggesting that it becomes dominated by direct collisional excitation.

8. Broad wings of $\mathrm{H} \alpha$ may be due in part to electron scattering, but there also appears to be an underlying broad emission component, seen almost exclusively at blueshifted speeds up to about $-7500 \mathrm{~km} \mathrm{~s}^{-1}$ (Fig. 8). This broad component appears sometime after day 41, is seen on days 64 through 95, and disappears again at very late times. We propose that this feature corresponds to the outermost parts of the SN ejecta that have almost reached the reverse shock (see Fig. 16). Material traveling at this speed would, in fact, just about reach the radius of the reverse shock by this time after explosion (Fig. 15). The broad features are also seen in $\mathrm{P}$ Cygni absorption in He I $\lambda 5876$ and O I $\lambda 7774$. The absorption requires some additional background continuum light source, which is likely to be the diffusion of radiation from the inner SN ejecta deposited by shock energy or radioactive decay. The luminosity required for the absorption strength implies that the underlying SN was overluminous as well, independent of CSM-interaction.

9. A possible explanation for why the broad features are seen only from day 64 through 95 is that before that time, the shocked shell was highly opaque (the broad features reside interior to the reverse shock; Fig. 16). Long after that time (by day 445), the SN ejecta luminosity has probably dropped far below that of the ongoing CSMinteraction region. 
10. The luminosity of the intermediate-width component of $\mathrm{H} \alpha$ is not correlated with the continuum luminosity of the SN (Fig. 12). It rises as the continuum luminosity fades. Compared to other SNe IIn, the $\mathrm{H} \alpha$ equivalent width is lower, but rises to similar values at late times more than 1 yr after explosion (Fig. 13). This is another clue that SN 2006tf has some additional source of continuum luminosity at early times, which is likely to be the slow diffusion of radiation from the massive sweptup opaque shell that mimics a normal H-recombination SN atmosphere, but at constant velocity.

11. The intermediate-width post-shock $\mathrm{H} \alpha$ emission has pronounced asymmetry at late times, showing an asymmetric and blueshifted profile at velocities within roughly $\pm 1000 \mathrm{~km} \mathrm{~s}^{-1}$. This may hint that dust formation has occurred in the dense post-shock cooling shell, analogous to SN 2006jc (Smith et al. 2008a), blocking gas mainly at the limbs and partially at the back side of the shell (bottom and top of the shell in Fig. 16).

12. One epoch of spectropolarimetry of SN 2006tf reveals relatively mild continuum polarization of $0.9 \%$ and also moderate depolarization of Balmer lines. Both effects are weaker than in other SNe IIn for which data are available, so we conclude that SN 2006tf shows no severe, global asymmetry. Thus, the simplified spherical scenario we describe next is basically valid.

\subsection{Conclusion: Optically Thick to Optically Thin}

Altogether, then, we conclude that available evidence requires a two-component model for the power source of SN 2006tf. These two components correspond to different regimes of CSM interaction: (1) shock interaction with extremely dense and opaque CSM with progenitor mass-loss rates of order $1 \mathrm{M}_{\odot} \mathrm{yr}^{-1}$, where the material has such high optical depth that the radiation generated by the shock interaction must diffuse out slowly through the massive amount of material after a delay corresponding to the diffusion timescale, and (2) more conventional, optically thin CSM interaction, where the luminosity generated by CSM interaction is able to escape without significant delay. As SN 2006tf expands and thins, it makes a transition between these two regimes.

Exactly how and when does this transition occur? In a normal SN II-P, the H-recombination photosphere recedes backward through the expanding SN ejecta, maintaining a roughly constant $R_{\mathrm{BB}}$ during the plateau phase as it passes through ejecta with a large gradient in expansion speed. The luminosity eventually drops sharply as the photosphere recedes all the way back through the $\mathrm{H}$ layer and the SN enters the nebular phase. This cannot be the case in SN 2006tf because the massive shell is expected to be geometrically thin and the observed expansion velocity is constant, while we observe no sudden transition akin to SNe II-P.

We propose that in SN 2006tf, the transition from an optically thick dense shell to the optically thin CSM interaction phase happened gradually as a result of clumping in the cold dense shell. The contact discontinuity, where material piles up between the forward and reverse shocks, will be severely Rayleigh-Taylor unstable and will not be a homogeneous constant-density spherical shell (Fig. 16, right panel). The size scale of density fluctuations will be similar to the thickness of the dense shell, which is geometrically very thin because it is relatively cold. Thus, the clumps that develop will have a typical size much smaller than the radius of the shell. The large number of small clumps across the surface of the shell will remain optically thick as recombination fronts move through them and their thermal energy is slowly radiated away, but the less dense regions between clumps will become optically thin more quickly, and the spacing between clumps will grow with time until all the material in the shell eventually becomes optically thin. Therefore, we would expect that the observed transition from effectively optically thick to effectively optically thin can appear to be a smooth transition, where the shrinking geometric covering factor of the optically thick clumps will mimic a shrinking average optical depth, while still allowing diffusion to be important for energy leakage from individual thick clumps. In effect, by looking through the increasingly porous shell, an observer can see the underlying SN ejecta even though the clumped material in the shell itself is still optically thick.

This decrease in the effective covering factor of opaque clumps (rather than an actual decrease in the radius) is the "dilution factor" $\zeta$ in Table 3 , needed to explain the gradual fading as the shell expands. Thus, diffusion of shock-deposited energy from an opaque envelope dominates at early times (as in SN 2006gy; Smith \& McCray 2007), but as time progresses, the subsequent ongoing CSM interaction remains constant and eventually comes to dominate the late-time luminosity and spectrum as the optically thick shell fades. This is why the $\mathrm{H} \alpha / \mathrm{H} \beta$ flux ratio appears to rise steadily.

At late times, this opaque shocked shell will have radiated away the bulk of its thermal energy and will cool significantly, heated only by the ongoing shock energy. The average densities in this $18 \mathrm{M}_{\odot}$ thin shell are extremely high - roughly $10^{11} \mathrm{~cm}^{-3}$ if the shell thickness is $\sim 10 \%$ of its radius. With the low luminosity and large radius at late times, it would not be surprising if dust formation occurs in the opaque cool shell, for which we see suggestive evidence in the late-time blueshifted $\mathrm{H} \alpha$ line profile. We encourage near-infrared spectroscopy of SN 2006tf in order to determine if it shows the expected $2.3 \mu \mathrm{m}$ CO bandhead emission, as was seen in SN 1998S (Gerardy et al. 2000; Fassia et al. 2001).

We might expect that this transition from thick to thin could, or should, happen for any SN IIn where the CSM close to the star is dense enough to be opaque, and this is probably true for other luminous SNe IIn at early times (e.g., SN 1994W; Chugai et al. 2004). The remarkable thing about SN 2006tf is just how high that luminosity was, how dense the CSM must have been, and how long the optically thick phase persisted. The wind density parameter, $w$, for SN 2006tf is orders of magnitude higher than that of most other SNe IIn. This is attributable to the very high mass-loss rate of the progenitor of SN 2006tf, but also to the fact that its wind speed of $190 \mathrm{~km} \mathrm{~s}^{-1}$ is relatively slow (SN $1994 \mathrm{~W}$, for example, apparently had a CSM speed around 800-1000 $\mathrm{km} \mathrm{s}^{-1}$; Chugai et al. 2004). One can therefore also understand the long duration of SN 2006tf in the context of its CSM speed: the terminal speed of $2000 \mathrm{~km} \mathrm{~s}^{-1}$ reached by the post-shock shell of SN 2006tf is still much greater than the $190 \mathrm{~km} \mathrm{~s}^{-1}$ speed of its CSM. In other SNe IIn with faster CSM, one might expect the interaction luminos- 
ity to drop as the shock decelerates, because the smaller difference in speed yields a weaker shock.

The mass-loss rates of $1-2 \mathrm{M}_{\odot} \mathrm{yr}^{-1}$ for about $10 \mathrm{yr}$ before core collapse, and $0.1-0.2 \mathrm{M}_{\odot} \mathrm{yr}^{-1}$ for several decades before that, are truly astounding. The only stars known to be capable of producing it are very massive LBVs during their so-called "giant eruptions" (Smith \& Owocki 2006). Normal stellar winds, even those of very massive and luminous stars, do not even come close. Thus, SN 2006tf adds a well established and rather extreme case to the growing body of evidence requiring massive eruptions in the decades preceding the core collapse of an initially very massive star. For SN 2006tf, the kinetic energy of that ejection was about $10^{48.8} \mathrm{ergs}$ (assuming $18 \mathrm{M}_{\odot}$ moving at $190 \mathrm{~km} \mathrm{~s}^{-1}$ ). In both SN progenitors and LBVs, these eruptions have no accepted explanation. For the SN progenitors, one potential origin comes from the pulsational pair instability discussed by Woosley et al. (2007) for SN 2006gy. That hypothesis, though, requires that the initial masses of these stars be $100-150 \mathrm{M}_{\odot}$, so any scenario would require that both SNe 2006gy and 2006tf must have marked the deaths of very massive stars.

The type of diffusion model we suggest provides an explanation for one extremely important aspect of SN 2006tf where a traditional CSM-interaction model fails: the blast-wave speed of SN 2006tf remains steady at a surprisingly slow value of $2000 \mathrm{~km} \mathrm{~s}^{-1}$, even from very early times only $32 \mathrm{~d}$ after discovery, despite the fact that it is radiating almost $10^{51} \mathrm{ergs}$ in visual light. Tra- ditional CSM interaction predicts an observable deceleration with time (e.g., Chugai \& Danziger 1994, 2003), while SNe 1988Z and 1998S provide observed examples where deceleration is clearly documented (Chugai \& Danziger 1994; Leonard et al. 2000; Pozzo et al. 2004). In the opaque shell-shocked model that we suggest for SN 2006tf, however, the bulk of the deceleration occurs very early as the blast wave runs through the opaque shell. Delayed photon diffusion allows the energy to be radiated away later. The massive circumstellar envelope that has been accelerated then coasts at a constant speed of $2000 \mathrm{~km} \mathrm{~s}^{-1}$ (so $R / R_{0}$ is not large and adiabatic losses are minimized) while its thermal energy is radiated away as the shell expands, thins, and cools.

We thank an anonymous referee for helpful comments. Some of the data presented herein were obtained at the W. M. Keck Observatory, which is operated as a scientific partnership among the California Institute of Technology, the University of California, and the National Aeronautics and Space Administration (NASA). The Observatory was made possible by the generous financial support of the W.M. Keck Foundation. We wish to extend special thanks to those of Hawaiian ancestry on whose sacred mountain we are privileged to be guests. KAIT was constructed and supported by donations from Sun Microsystems, Inc., the Hewlett-Packard Company, AutoScope Corporation, Lick Observatory, the US National Science Foundation (NSF), the University of California, the Sylvia \& Jim Katzman Foundation, and the TABASGO Foundation. A.V.F.'s supernova group at U.C. Berkeley is supported by NSF grant AST-0607485 and by the TABASGO Foundation. Research by A.J.B. is supported by NSF grant AST-0548198. We thank Misty Bentz for assistance with the Keck/ESI observing run.

\section{REFERENCES}

Aldering, G., et al. 2006, ApJ, 650, 510

Aretxaga, I., et al. 1999, MNRAS, 309, 343

Barkat, Z., Rakavy, G., \& Sack, N. 1967, Phys. Rev. Lett., 18, 379

Benetti, S., Cappellaro, E., Danziger, I. J., Turatto, M., Patat, F., \& Della Valle, M. 1998, MNRAS, 294, 448

Benetti, S., Cappellaro, E., Turatto, M., Taubenberger, S. Harutyunyan, A., \& Valenti, S. 2006, ApJ, 654, L129

Bond, J. R., Arnett, W. D., \& Carr, B. J. 1984, ApJ, 280, 825

Branch, D., Falk, S. W., McCall, M. L., Rybski, P., Uomoto, A. K., \& Wills, B. J. 1981, ApJ, 244, 780

Chevalier, R. A., \& Fransson, C. 1994, ApJ, 420, 268

Chugai, N. N., \& Danziger, I. J. 1994, MNRAS, 268, 173

Chugai, N. N., \& Danziger, I. J. 2003, Ast. Lett., 29, 649

Chugai, N. N., et al. 2004, MNRAS, 352, 1213

Faber, S. M., et al. 2003, Proc. SPIE, 4841, 1657

Falk, S. W., \& Arnett, W. D. 1973, ApJ, 180, L65

Falk, S. W., \& Arnett, W. D. 1977, ApJS, 33, 515

Fassia, A., et al. 2000, MNRAS, 318, 1093

Fassia, A., et al. 2001, MNRAS, 325, 907

Filippenko, A. V. 1982, PASP, 94, 715

Filippenko, A. V. 1991, in SN 1987A and Other Supernovae, ed. I. J. Danziger \& K. Kjär (Garching: ESO), 343

Filippenko, A. V. 1997, ARAA, 35, 309

Filippenko, A. V. 2000, in Cosmic Explosions, ed. S. S. Holt \& W. W. Zhang (New York: AIP), 123

Filippenko, A. V., Li, W. D., Treffers, R. R., \& Modjaz, M. 2001, in Small-Telescope Astronomy on Global Scales, ed. W. P. Chen, C. Lemme, \& B. Paczyński (San Francisco: ASP, Conf. Ser. Vol. 246), 121

Galama, T. J., et al. 1998, Nature, 396, 670

Gal-Yam, A., et al. 2007, ApJ, 656, 372

Gerardy, C. L., Fesen, R. A., Höflich, P., \& Wheeler, J. C. 2000, AJ 119,2968

Germany, L. M., Reiss, D. J., Sadler, E. M., Schmidt, B. P., \& Stubbs, C. W. 2000, ApJ, 533, 320

Hamuy, M., et al. 2003, Nature, 424, 651

Hoffman, J. L., Leonard, D. C., Chornock, R., Filippenko, A. V., Barth, A. J., \& Matheson, T. 2008, ApJ, submitted, astro-ph/0709.3258

Kotak, R., Meikle, W. P. S., Adamson, A., \& Leggett, S. K. 2004, MNRAS, 354, L13

Leibundgut, B., Kirshner, R. P., Pinto, P. A., Rupen, M. P., Smith, R. C., Gunn, J. E., \& Schneider, D. P. 1991, ApJ, 372, 531
Leonard, D. C., Filippenko, A. V., Barth, A. J., \& Matheson, T. 2000, ApJ, 536, 239

Leonard, D. C., Filippenko, A. V., Ardila, D. R., \& Brotherton, M. S. 2001, ApJ, 553, 861

Leonard, D. C., et al. 2002, PASP, 114, 35

Miller, J. S., Robinson, L. B., \& Goodrich, R. W. 1988, in Instrumentation for Ground-Based Astronomy, ed. L. B. Robinson (New York: Springer-Verlag), 157

Ofek, E. O., et al. 2007, ApJ, 659, L13

Oke, J. B., et al. 1995, PASP, 107, 375

Owocki, S. P., Gayley, K. G., \& Shaviv, N. J. 2004, ApJ, 616, 525

Pastorello, A., et al. 2002, MNRAS, 333, 27

Pozzo, M., et al. 2004, MNRAS, 352, 457

Prieto, J., et al. 2007, arXiv:0706.4088

Quimby, R. M., Aldering, G., Wheeler, J. C., Höflich, P., Akerlof, C. W., \& Rykoff, E. S. 2007a, ApJ, 668, L99

Quimby, R., Castro, F., Mondol, P., Caldwell, J., \& Terrazas, E. 2007b, CBET, 793, 1

Rakavy, G., \& Shaviv, G. 1967, ApJ, 148, 803

Raymond, J. C., et al. 2007, ApJ, 659, 1257

Rigon, L., et al. 2003, MNRAS, 340, 191

Schlegel, D. J., Finkbeiner, D. P., \& Davis, M. 1998, ApJ, 500, 525

Schlegel, E. M. 1990, MNRAS, 244, 269

Schmidt, G. D., Elston, R., \& Lupie, O. L. 1992, AJ, 104, 1563

Sheinis, A. I., et al. 2002, PASP, 114, 851

Smith, N. 2008, in IAU Symposium 250: Massive Stars as Cosmic Engines, F. Bresolin, P.A. Crowther, \& J. Puls, eds. (Cambridge Univ. Press), 193

Smith, N., \& Hartigan, P. 2006, ApJ, 638, 1045

Smith, N., \& McCray, R. 2007, ApJ, 671, L17

Smith, N., \& Owocki, S. P. 2006, ApJ, 645, L45

Smith, N., et al. 2003, AJ, 125, 1458

Smith, N., Foley, R. J., \& Filippenko, A. V. 2008a, ApJ, 680, 568

Smith, N., et al. 2008b, ApJ, in press (arXiv:0802.1743)

Smith, N., Li, W., Foley, R. J., Wheeler, J. C., Pooley, D., Chornock, R., Filippenko, A. V., Silverman, J. M., Quimby, R., Bloom, J., \& Hansen, C. 2007, ApJ, 666, 1116

Sollerman, J., Cumming, R. J., \& Lundqvist, P. 1998, ApJ, 493, 933

Stathakis, R. A., \& Sadler, E. M. 1991, MNRAS, 250, 786

Turatto, M., et al. 1993, MNRAS, 262, 128

Turatto, M., et al. 2000, ApJ, 534, L57

Wang, L., Wheeler, J. C., Li, Z., \& Clocchiatti, A. 1996, ApJ, 467, 435 
Wang, L., Howell, D. A., Höflich, P., \& Wheeler, J. C. 2001, ApJ, 550, 1030

Wang, L., Baade, D., Höflich, P., Wheeler, J. C., Kowabata, K., \& Nomoto, K. 2004, ApJ, 604, L53
Wood-Vasey, W. M., Wang, L., \& Aldering, G. 2004, ApJ, 616, 359 Woosley, S. E., Blinnikov, S., \& Heger, A. 2007, Nature, 450, 390 